\title{
ANALISIS DE PARAMETROS OCEANOGRAFICOS Y ATMOSFERICOS DEL SENO DE RELONCAVI
}

\section{ANALYSIS OF OCEANOGRAPHIC AND ATMOSPHERIC PARAMETERS OF SENO DE RELONCAVI}

\author{
Luis Soto-Mardones ${ }^{1}$, Jaime Letelier ${ }_{4}^{2,3}$ \\ Sergio Salinas ${ }^{2}$, E. Pinillas ${ }^{4}$ y Juan P. Belmar ${ }^{5}$ \\ 'Facultad de Ciencias, Departamento de Física, Universidad de Bío-Bío, \\ Av. Collao 1202, casilla 5-C, CP 4081112 Concepción, Chile. \\ ${ }^{2}$ Escuela de Ciencias del mar, Facultad de recursos naturales, \\ ${ }_{3}$ Pontificia Universidad de Católica de Valparaíso, Av. Altamirano 1480, Valparaíso, Chile. \\ ${ }^{3}$ Sección de Oceanografía Instituto de Fomento Pesquero, Av. Blanco 839, Valparaíso, Chile. \\ ${ }^{4}$ Sección de predicción del oleaje y la corrientes INTESAL. \\ ${ }^{5}$ Servicio Hidrográfico y Oceanográfico de la Armada, Errázuriz 254, Playa Ancha, Valparaíso.
}

\section{RESUMEN}

La recopilación de información histórica, tales como mediciones de corrientes, oleaje y viento no ha sido suficiente para describir la dinámica del seno Reloncaví. Sin embargo, a pesar de la información parcelada, las altas magnitudes de corrientes en las zonas de Chaica, Quillaipe y El Paso Nao nos permiten inducir que las corrientes baroclínicas modulan fuertemente la dinámica de la cuenca. Por otro lado, los vientos mostraron una alta variabilidad estacional y son modulados por la línea de costa, siendo estos en parte responsables de la circulación y el oleaje superficial. Además, la circulación superficial en los primeros $10 \mathrm{~m}$ esta fuertemente modulada por el ingreso de aguas dulce del estuario de Reloncaví. Además, la máxima amplitud del nivel del mar es de $7.64 \mathrm{~m}$, con una amplitud promedio de $7.46 \mathrm{~m}$, y donde la mayor parte de la variabilidad del nivel del mar esta asociada a la marea.

Finalmente, para describir el comportamiento del oleaje se comparan el método empírico de Massel con el modelo numérico del Mike21. Resultando que el clima de ola estaría siendo modificado por la gran variabilidad espacial y temporal de la magnitud y dirección de las corrientes de la onda de marea

Palabras claves: Baroclínicas, Olas, Pluma, Reloncaví, Viento

\section{ABSTRACT}

The historical information compilation of currents, waves, and wind measurements has not been enough to appropriately describe the dynamics of Seno de Reloncaví. However, in spite of the low diversity of studies, the high current magnitudes registered at Chaica, Quillaipe and Paso Nao allow us to infer baroclinic currents strongly modulate the basin dynamics. Furthermore, the wind showed a high seasonal variability, and it is modulated by the coastline, thus being somehow responsible of the surface circulation and waves. In addition, the surface circulation $(\sim 10 \mathrm{~m})$ is strongly modulated by freshwater input coming from Estuario de Reloncaví. Further, the maximum sea level amplitude was 7.64 m, with 7.46 $\mathrm{m}$ as an average, where the major sea level variability is linked to tide.

Finally, in order to describe the wave behavior, the results of the empiric method of Massel and the numerical model of Mike 21 are compared. As a result, the wave behavior would be modified by the high spatial and temporal variability of magnitude and direction of the tidal wave.

KEYwords: Baroclinic, Waves, Feather, Reloncavi, Wind 


\section{INTRODUCCION}

El seno de Reloncaví es un sistema semicerrado rodeado de una línea de costa casi circular que se conecta por el este con el estuario de Reloncaví y al sur con el Golfo de Ancud a través de tres pasos (Tautil, Queullin y Nao) que lo convierten en un laboratorio natural de mesoescala. El seno es el inicio de una de las mayores extensiones de canales, fiordos, senos y golfos interiores superando los $84000 \mathrm{~km}$ de costa en una extensión meridional de solo $1000 \mathrm{~km}$. Estas estructuras geográficas tienen su origen en la acción de la erosión glaciar y el hundimiento del valle central debido a la actividad tectónica de la zona (Borgel, 1970-1971). En su interior se desarrolla un sin número de sistemas estuarinos locales producto de la interacción de aguas salinas oceánicas y aguas dulces provenientes de aportes de lluvias, ríos, glaciares y ventisqueros (Silva et a., 1998; Guzmán y Silva, 2002; Cáceres et al., 2002; Cáceres et al., 2003; Cáceres, 2004;
Vera, 2005; Silva \& Palma, 2006; Valle-Levinson et al., 2007). Todos estos factores determinan que la dinámica de las corrientes de la zona de los canales este modulada por la influencia de la marea, los flujos salientes y entrantes desde los estuarios y fiordos (capa superior e inferior, respectivamente), como también desde los canales y pasos hacia el océano. Por otro lado, el oleaje en el mar interior estaría controlado por condiciones locales más que por perturbaciones oceánicas que pudieran entrar por los grandes canales que conectan con el océano como el canal Chacao y la boca del Guafo.

El presente trabajo consistió fundamentalmente en la recolección y análisis de datos disponibles de parámetros batimétricos, oceanográficos y atmosféricos, a partir de la literatura científica, instituciones y empresas que han realizado mediciones en la zona. A pesar de la escasa información disponible, es posible describir a grandes rasgos la dinámica que domina seno de Reloncaví.

TABLA I. Recolección de Parámetros Topográficos, Oceanográficos y Atmosféricos.

TABLE I. Topographic, oceanographic and atmospheric parameters compilation.

\begin{tabular}{|c|c|c|c|c|}
\hline \multirow[t]{2}{*}{ Parámetros } & \multicolumn{2}{|c|}{ Localización } & \multirow[t]{2}{*}{ Periodos } & \multirow[t]{2}{*}{ Fuentes } \\
\hline & Latitud (S) & Longitud W) & & \\
\hline Batimetría & $41^{\circ} 26^{\prime}-42^{\circ}$ & $73^{\circ} 45^{\prime}-72^{\circ} 30^{\prime}$ & & CIMAR \\
\hline Temperatura & \multirow{3}{*}{\multicolumn{2}{|c|}{$\begin{array}{l}\text { Estaciones } 1 \text { a } 4, \\
\text { dentro del seno } \\
\text { (Silva y Palma, 2006) }\end{array}$}} & $1 / 3 / 70-30 / 1 / 70$ & CIMAR \\
\hline Salinidad & & & $1 / 3 / 70-30 / 1 / 70$ & CIMAR \\
\hline Oxigeno & & & $1 / 3 / 70-30 / 1 / 70$ & CIMAR \\
\hline Nivel del Mar & $41^{\circ} 29^{\prime} 05^{\prime \prime}$ & $72^{\circ} 57^{\prime} 39^{\prime \prime}$ & 1990-2006 & SHOA \\
\hline Viento de Puerto Montt & $41^{\circ} 30^{\prime}$ & $72^{\circ} 54^{\prime}$ & $1 / 1 / 06-30 / 9 / 08$ & $\begin{array}{c}\text { Servicio Meteorológico de la } \\
\text { Armada }\end{array}$ \\
\hline Correntómetros Chaicas & $41^{\circ} 36^{\prime} 32^{\prime \prime}$ & $72^{\circ} 37^{\prime} 16^{\prime \prime}$ & $\begin{array}{l}20 / 3 / 06-5 / 5 / 06 \\
4 / 7 / 06-18 / 8 / 06\end{array}$ & Pesquera CAMANCHACA \\
\hline Correntómetros Quillaipe & $41^{\circ} 31^{\prime} 30^{\prime \prime}$ & $72^{\circ} 48^{\prime} 15^{\prime \prime}$ & $\begin{array}{l}\text { 20/3/06- } \\
5 / 5 / 06\end{array}$ & \\
\hline ADCP Paso Nao & $41^{\circ} 53,4^{\prime}$ & $72^{\circ} 49,2^{\prime}$ & $\begin{array}{l}11 / 10 / 04- \\
12 / 10 / 04\end{array}$ & CIMAR \\
\hline Modelo Empírico MASSEL & $41^{\circ} 26^{\prime}-42^{\circ}$ & $73^{\circ} 45^{\prime}-72^{\circ} 30^{\prime}$ & & \\
\hline Modelo Numérico MIKE21 & $41^{\circ} 26^{\prime}-42^{\circ}$ & $73^{\circ} 45^{\prime}-72^{\circ} 30^{\prime}$ & & INTESAL \\
\hline
\end{tabular}




\section{MATERIALES Y METODOS}

La información recopilada aparece detallada en el Tabla I, donde destaca la batimetría de alta resolución la cual fue compilada y grillada por Rodrigo (2006). Las distribuciones de parámetros oceanográficos de temperatura, salinidad y oxígeno de las zonas fueron reportadas por Pickard $(1971,1973)$ del crucero Hudson 1970. Además, se analizaron 17 años de datos diarios de nivel del mar de la estación mareográfica de Puerto Montt que fueron proporcionados por el Servicio Hidrográfico y Oceanográfico de la Armada (SHOA), mientras que el Servicio Meteorológico de la Armada proporcionó dos años de viento cada 3 horas de la estación Meteorológica de la misma ciudad. Por otro lado, la empresa Patagonia en Chaicas $\left(41^{\circ} 40^{\prime} \mathrm{S}-72^{\circ} 39^{\prime} \mathrm{W}\right)$ y Quillaipe $\left(41^{\circ} 34^{\prime} \mathrm{S}\right.$ - 72 $44^{\prime}$ W) instaló tres correntómetros en tres profundidades $(5,10$ y $20 \mathrm{~m})$ donde registró la magnitud y dirección de la corriente cada 5 minutos. La instalación de los correntómetros en Chaicas abarcó los periodos de otoño e invierno del 2006, mientras que las mediciones en Quillaipe abarcaron el periodo de otoño 2006. Además, se analizaron mediciones de corrientes realizadas con un equipo Acoustic Doppler Current Profiler (ADCP) en un circuito cerrado de 2 días de noviembre de 2004 en el Paso Nao, como parte del crucero Cimar Fiordo 10. Finalmente, se realizaron estimaciones de oleaje y corrientes mediante el modelo empírico de Massel y el modelo numérico Mike21 proporcionados por la empresa INTESAL.

\section{RESULTADOS}

Hidrografía: el transecto de temperatura, salinidad y oxígeno reportado por Silva et al., 1995 mediante un reanálisis de los trabajos de Pickard (1971, 1973), muestran claramente una pluma somera calida $\left(>13^{\circ} \mathrm{C}\right)$, de baja salinidad $(\sim 30 \%$ o) y de alta concentración de oxígeno (6 a $7.5 \mathrm{ml} / \mathrm{l})$. Los parámetros hidrográficos muestran una dinámica estuarina dominada por el aporte de agua dulce proveniente del estuario de Reloncaví, mientras que en profundidad es modulada por aguas de origen oceánica, es decir, la temperatura es menor a $11^{\circ} \mathrm{C}$, los índices de salinidad son superiores que en superficie (>32.5\%o) y el oxigeno es aproximadamente es menor $4 \mathrm{ml} / \mathrm{l}$.

Nivel del Mar: dentro del contexto de los forzantes de la circulación en la zona de los canales, la marea es uno de los factores más importantes en la zona. Los registros horarios y diarios del nivel del mar (NM) muestran una amplitud máxima de $7.64 \mathrm{~m}$ y una amplitud promedio de $7.46 \mathrm{~m}$ (Tabla II). Por otra parte, los resultados del NM diario (4.5 a 5.5 $\mathrm{m})$, con una amplitud máxima de $1 \mathrm{~m}$, permiten concluir que la mayor parte de la variabilidad en el registro de Puerto Montt está asociada principalmente a la marea. La máxima amplitud del NM aparece en dos fases anuales Marzo-Abril y Julio-Agosto. El promedio total de amplitud para un periodo de 15 año fue de $7.45 \mathrm{~m}$, mientras que la mínima amplitud $(7.26 \mathrm{~m})$ tiende a aparecer durante el mes de enero.

Tabla II. Estadística básica de valores de altura de nivel del mar y la amplitud en la estación costera de Puerto Montt (Fuente: Servicio Hidrográfico y Oceanográfico de la Armada de Chile).

Table II. Basic statistics of sea level, and amplitude at the coastal station of Puerto Montt (Source: Servicio Hidrográfico y Oceanográfico de la Armada de Chile).

\begin{tabular}{lccccccccccccc}
\hline $\mathbf{m})$ & Ene & Feb & Mar & Abr & May & Jun & Jul & Ago & Sep & Oct & Nov & Dic & Tot \\
\hline Promedio & 4.83 & 4.83 & 4.83 & 4.83 & 4.85 & 4.89 & 4.87 & 4.85 & 4.82 & 4.82 & 4.82 & 4.83 & 4.84 \\
Máximo & 8.64 & 8.61 & 8.59 & 8.80 & 8.58 & 8.51 & 8.51 & 8.63 & 8.48 & 8.64 & 8.53 & 8.53 & 8.80 \\
Mínimo & 1.38 & 1.15 & 1.07 & 1.18 & 1.33 & 1.12 & 0.94 & 1.01 & 1.00 & 1.15 & 1.20 & 1.20 & 0.94 \\
Amplitud & 7.26 & 7.46 & 7.52 & 7.62 & 7.25 & 7.39 & 7.57 & 7.62 & 7.48 & 7.49 & 7.33 & 7.33 & 7.45 \\
\hline
\end{tabular}


En términos relativos, el registro de nivel del mar muestra que las observaciones se concentraron $(>80 \%)$ entre $3.50 \mathrm{~m}$ y $6.50 \mathrm{~m}$ de altura. Por otro lado, menos de un $2.5 \%$ de las observaciones representan a observaciones del nivel del mar que sobrepasan los 8 $\mathrm{m}$, y estos corresponden a la combinación de las mareas más altas con la presencia de frentes de mal tiempo. En el dominio de las frecuencias de los datos horarios, la figura 1 muestra picos significativos asociados a las 24, 12, 6 y 4 hrs, resultando que la mayor parte de la variabilidad del NM está asociado a fluctuaciones de periodos menores a un día. Los períodos de 24 y $12 \mathrm{hrs}$ representan un régimen de marea semidiurno mixto, dos pleamares y dos bajamares diarias de significativa diferencia amplitud entre ellas. Los picos de 6 y 4 horas son componentes de agua somera de la marea que se potencian en aguas someras.

En el dominio de las frecuencias se determinó que la mayor parte de la variabilidad del NM está asociada a fluctuaciones de periodos menores a un día. Y la distribución general de valores extremos, indica que para un periodo de retorno de 10 años, la probabilidad es de un $10 \%$ y el NM es $\sim 8.62 \mathrm{~m}$; para un periodo de 20 años, la probabilidad de un 5 $\%$ es posible que ocurra un NM de $8.72 \mathrm{~m}$. Con una probabilidad de un $2 \%$ cada 50 años ocurre un NM de $8.77 \mathrm{~m}$ y con una probabilidad de $1 \%$ cada 100 años podría generarse un NM de $8.80 \mathrm{~m}$.

Viento: por las características topográficas del seno de Reloncaví la influencia de los vientos locales serían determinante en la estimación del oleaje y en la circulación superficial. En la estación meteorológica ubicada en Puerto Montt, el viento entre 2007 y 2008 presentó una alta variabilidad (Fig. 2), con fluctuaciones diarias, semanales y estacionales. La estacionalidad de la componente norte-sur (v) se observa claramente en el dominio del viento desde el norte, noroeste (abril y noviembre), y el dominio de la componente desde el sur (noviembre y abril). Además, en el dominio de las frecuencias de las direcciones del viento, se encontró que predominó en orden descendente: la combinación de viento sur $(i \%)$ y suroeste $(i \%)$, le sigue dirección norte $(i \%)$ y oeste $(i \%)$ entre un rango de $22 \%$ a $9 \%$ de los registros. Por otro lado, las magnitudes presentadas muestran que la mayor frecuencia de eventos de viento se concentró entre los rangos de 1 a $3 \mathrm{~m} / \mathrm{s}$ (40.9\%), de 3 a 6 m/s (34.6\%), mientras entre 6 a 9 $\mathrm{m} / \mathrm{s}$ se presentaron el $4 \%$ de los eventos y mas de 9 $\mathrm{m} / \mathrm{s}$ solamente el $0.16 \%$, mientras que calmas representan un $20.1 \%$ de los registros.

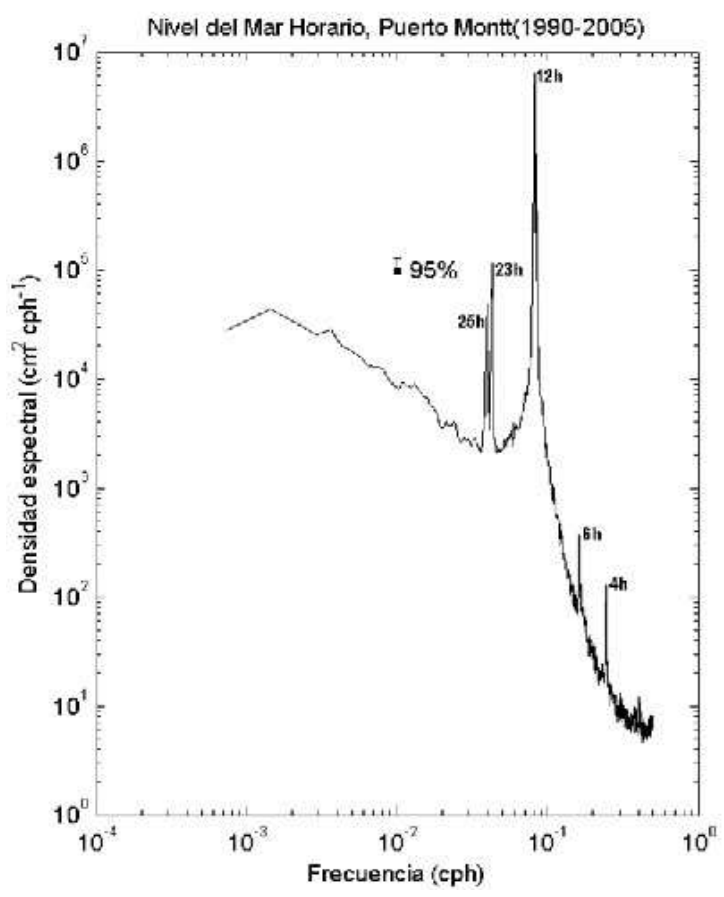

FIgURA 1. Espectro de frecuencia de datos de nivel del mar horarios de Puerto Montt que abarcan desde 1990 a 2005.

FIGURE 1. Frequency spectrum of hourly sea level data in Puerto Montt from 1990 up to 2005. 
Análisis oceanográficos y atmosféricos: Soto-MARDOnES, L. ET AL.
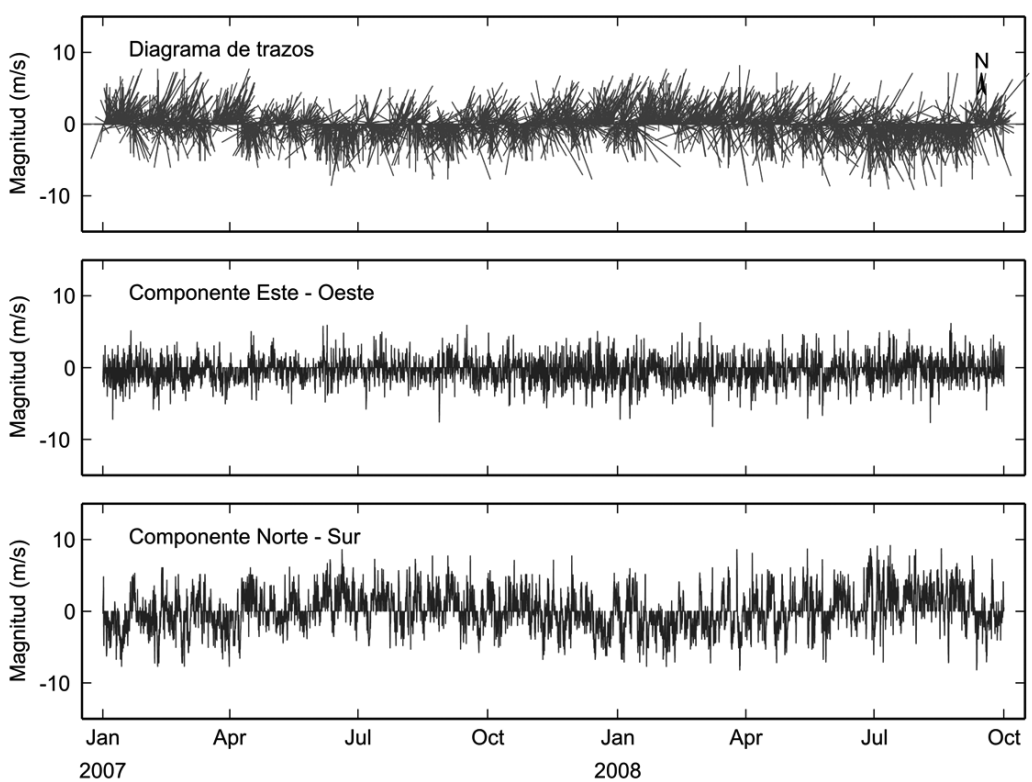

Figura 2. Diagrama de trazos y las componentes u (Este-Oeste) y v (Norte-Sur) del viento en Puerto Montt.

FIGURE 2. Vector plot and its wind components u (east-west) and v (north-south) in Puerto Montt.

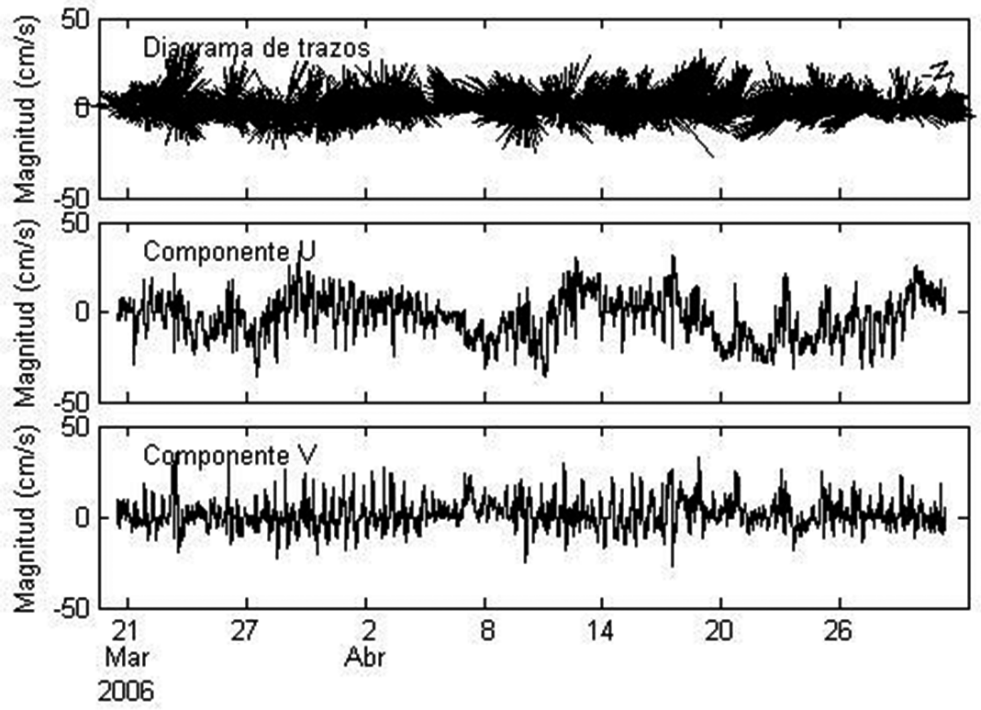

Figura 3. Diagrama de trazos y las componentes de velocidades U y V de instalación Chaicas (5m) para el periodo de otoño.

FIGURE 3. Vector plot and its velocity components $u$ and $v$ at Chaicas $(5 \mathrm{~m})$ for autumn season. 
Corriente: debido a las escasas mediciones de corrientes en el seno de Reloncaví, los anclajes de las estaciones de Chaicas y Quillaipe resultan una información valiosa. Las posiciones de los anclajes están indicadas en la Tabla I, cada instalación contiene tres correntómetros, ubicados en tres profundidades $(5,10$ y $20 \mathrm{~m})$. A $5 \mathrm{~m}$, se muestra una alta variabilidad mediante el diagrama de trazos y las componentes $\mathrm{U}$ y $\mathrm{V}$ fueron rotadas siguiendo el eje de máxima varianza $\left(\sim 70^{\circ}\right)$. Así, la máxima variabilidad es mostrada a lo largo (V) y perpendicular a la costa (U), donde las componentes presentan una señal diurna y semidiurna que varia en amplitud dependiendo del periodo del registro. La componente $\mathrm{U}$ presenta periodos de variación de entre 11 y 12 días. Además el máximo valor individual correspondió a $36,96 \mathrm{~cm} / \mathrm{s}$ en dirección NW.

A $10 \mathrm{~m}$ de profundidad, el instrumento presentó anomalías que no permitieron determinar la intensidad y dirección de las corrientes. Por otro lado, a $20 \mathrm{~m}$ de profundidad (Fig. 4), la intensidad de las corrientes es relativamente menor que en la capa superficial, además, las perturbaciones semidiurnas son mucho menores que las observadas en superficie. A pesar de lo anterior, aparece un evento importante que perturba la serie (entre el 17 y 22 de abril) con corrientes mayores a $10 \mathrm{~cm} / \mathrm{s}$ aparecen a lo largo y fuera de la costa en dirección noroeste (NW).

Las frecuencias en las direcciones de corriente mostró un flujo relativamente variable donde la dirección predominante fue la combinación $\mathrm{NW}(21,54 \%)$ y $\mathrm{N}(14,91 \%)$ sobre las componentes $\mathrm{S}(13,05 \%)$ y $\mathrm{SE}$ $(11,71 \%)$ que la siguen en importancia.

El segundo conjunto de mediciones de la estación de Chaicas se realizó durante el período de invierno, cuyos resultados (Fig. 5) mostraron mayor intensidad y variabilidad que las corrientes observadas durante la época de otoño. De hecho, entre el 5 y 26 de julio, las intensidades sobrepasan los $20 \mathrm{~cm} / \mathrm{s}$ con pulsos cortos combinados de más de $40 \mathrm{~cm} / \mathrm{s}$ con dirección S y NW. Esta variabilidad se repite a partir de 6 de agosto donde predominaron flujos hacia fuera de la costa.

FIGURA 4. Diagrama de trazos y las componentes de velocidades U y V de instalación Chaicas (20m) para el periodo de otoño.

FIGURE 4 . Vector plot and its velocity components $u$ and $v$ at Chaicas $(20 \mathrm{~m})$ for autumn season. 
Análisis oceanográficos y atmosféricos: Soto-MARDONES, L. ET AL.

Figura 5. Diagrama de trazos y las componentes de velocidades U y V de instalación Chaicas (5m) para el periodo de invierno.

FIGURE 5. Vector plot, and its velocity components $u$ and v $(5 \mathrm{~m})$ for winter season.

Las frecuencias en las direcciones de las corrientes variaron desde S $(24,7 \%)$, SE $(163,15 \%)$ y NW $(19.81 \%)$ con el resto de las direcciones con porcentajes menores a $11 \%$. Los máximos individuales observados a esta profundidad superan los $63 \mathrm{~cm} / \mathrm{s}$ en dirección N y NW.

A $10 \mathrm{~m}$ de profundidad, los registros se observaron intensas corrientes $(>20 \mathrm{~cm} / \mathrm{s})$ con una fuerte componente perpendicular a la costa y una variabilidad en ambas componentes asociadas al ciclos diurnos y semidiurnos. Por otro lado, resaltan las corrientes a $20 \mathrm{~m}$ de profundidad, donde las magnitudes son superiores a las reportadas en otoño, con flujos significativos $(>15 \mathrm{~cm} / \mathrm{s})$ y pulsos de corrientes intensas $(>30 \mathrm{~cm} / \mathrm{s})$ a lo largo y perpendiculares a la costa.

Las mediciones realizadas en Quillaipe durante otoño muestran una gran variabilidad en dirección y magnitud. A la profundidad de $5 \mathrm{~m}$, las corrientes alcanzan magnitudes del orden de los $40 \mathrm{~cm} / \mathrm{s}$, con un promedio aproximado de $10 \mathrm{~cm} / \mathrm{s}$. A la profundidad de $10 \mathrm{~m}$ las corrientes son más débiles que en superficie y las máximas alcanzan magnitudes del orden de 1 os $30 \mathrm{~cm} / \mathrm{s}$, con un promedio aproximado de $8 \mathrm{~cm} / \mathrm{s}$. Y finalmente, a la profundidad de $20 \mathrm{~m}$, las corrientes son relativamente más débiles que a $10 \mathrm{~m}$ de profundidad y los máximos registros alcanzan magnitudes del orden de los $25 \mathrm{~cm} / \mathrm{s}$, con un promedio aproximado de $8 \mathrm{~cm} / \mathrm{s}$. Las observaciones muestran que las corrientes en todas las profundidades en este sector son fuertemente afectadas por la onda de marea diurna y semidiurna. Resalta que las direcciones dominantes en las tres profundidades son hacia el NW $\left(320^{\circ}\right)$ y SE $\left(140^{\circ}\right)$. Otra información valiosa que se logró rescatar, son las mediciones realizadas con ADCP en el paso Nao, las que a pesar del corto periodo de muestreo (2 días), nos permite dar una visión global de la dinámica de la corrientes que atraviesan hacia el interior y exterior del seno, y permiten justificar la alta variabilidad que se observa en el campo de vientos, altura nivel del mar y los anclajes de Chaicas y Quillaipe. La figura 6 se muestra un circuito realizado cada tres horas por las pasadas por el transecto 


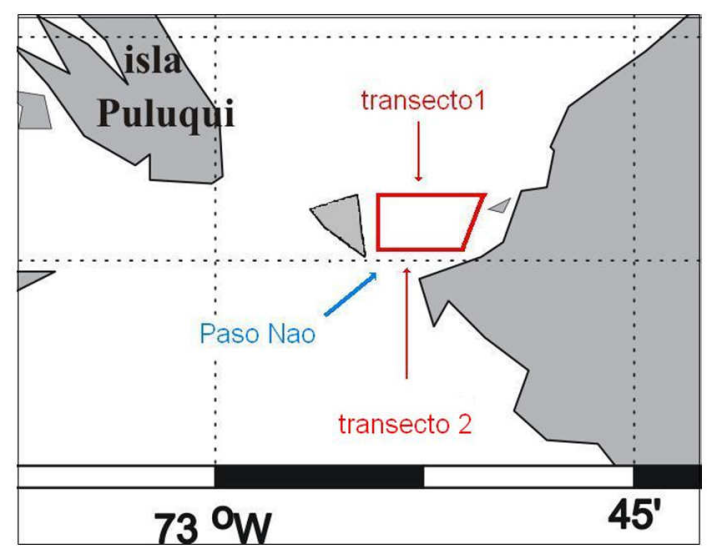

FIGURA 6. Muestreo con ADCP en el proyecto CIMAR 10 FIORDO, durante el periodo del 13 y 14 de noviembre del 2004.

FIGURE 6. ADCP measurements during CIMAR 10 FIORDO project, (November 13rd-14th, 2004).

Las figura 7 muestran las seis pasadas por el transecto 1 , donde se puede observar claramente en cada repetición del mismo recorrido ( $\sim 3 \mathrm{hrs})$ como el campo de distribución vertical de velocidad se va modificando. Por el borde oriental del Paso Nao, se observó una pluma somera (11:42 hrs) saliendo del seno de Reloncaví con velocidades de $10 \mathrm{~cm} / \mathrm{s}$ y un robusto núcleo se desplaza hacia el norte con velocidad sobre los $30 \mathrm{~cm} / \mathrm{s}$, posteriormente una columna hacia el sur desde la superficie hasta el fondo y una contracorriente pegada al borde occidental. $\mathrm{Al}$ cabo de tres horas (14:20 hrs) el esquema de circulación cambió radicalmente, donde el sector del borde occidental se desplazó hacia el norte y el borde oriental hacia el lado sur, además, se observó una intensificación de la pluma somera (> $30 \mathrm{~cm} / \mathrm{s})$. A las 16:13 hrs la circulación hacia el sur persistió, dominando casi toda el área del Paso Nao, posteriormente a las 01:23 del día 14 la circulación hacia el norte se fortaleció en el borde occidental alcanzando una velocidad en el centro de $40 \mathrm{~cm} / \mathrm{s}$, en cambio, en el borde oriental la circulación hacia el sur se presentó débil, aunque la pluma somera se expande hacia en oeste. A la 03:07 se observó una intensificación de la pluma somera alcanzando velocidades superiores a $30 \mathrm{~m} / \mathrm{s}$ en todo el ancho del paso Nao, mientras que la corriente hacia el norte - por el borde occidental- se debilitó ( $20 \mathrm{~cm} / \mathrm{s})$. A las 05:20 del segundo día, la circulación no mostró un patrón definido, aunque, persiste la intensificación de la pluma superficial (> $30 \mathrm{~cm} / \mathrm{s}$ )

Aunque no es posible comparar todos los parámetros (hidrografía, nivel del mar, viento, corrientes de anclajes) en forma temporal (Tabla I), queda en evidencia que estos confirman la alta variabilidad de la circulación superficial y en profundidad. Los datos de corrientes directas (Fig. 7) muestran en forma más convincente como las aguas del Seno de Reloncaví están fuertemente afectados por el forzante de la onda de marea y el desnivel de la superficie de agua causada por la acumulación de agua por efecto del viento que afecta a toda la columna de agua, por la circulación de tipo estuarino por el aporte de agua salobre proveniente del estuario de Reloncaví y por el efecto directo del viento sobre las capas superficiales de agua.

En este capitulo, de acuerdo a la recopilación de antecedentes, se analizan un modelo empírico y el otro numérico en ejecución en la zona de estudio. Para la aplicación de modelos numéricos en la cuenca semi-cerrada, somera y escasa influencia del océano abierto, las variables dinámicas que estarían modulando el oleaje serían: las corrientes marinas, marea, vientos y línea de costa. 

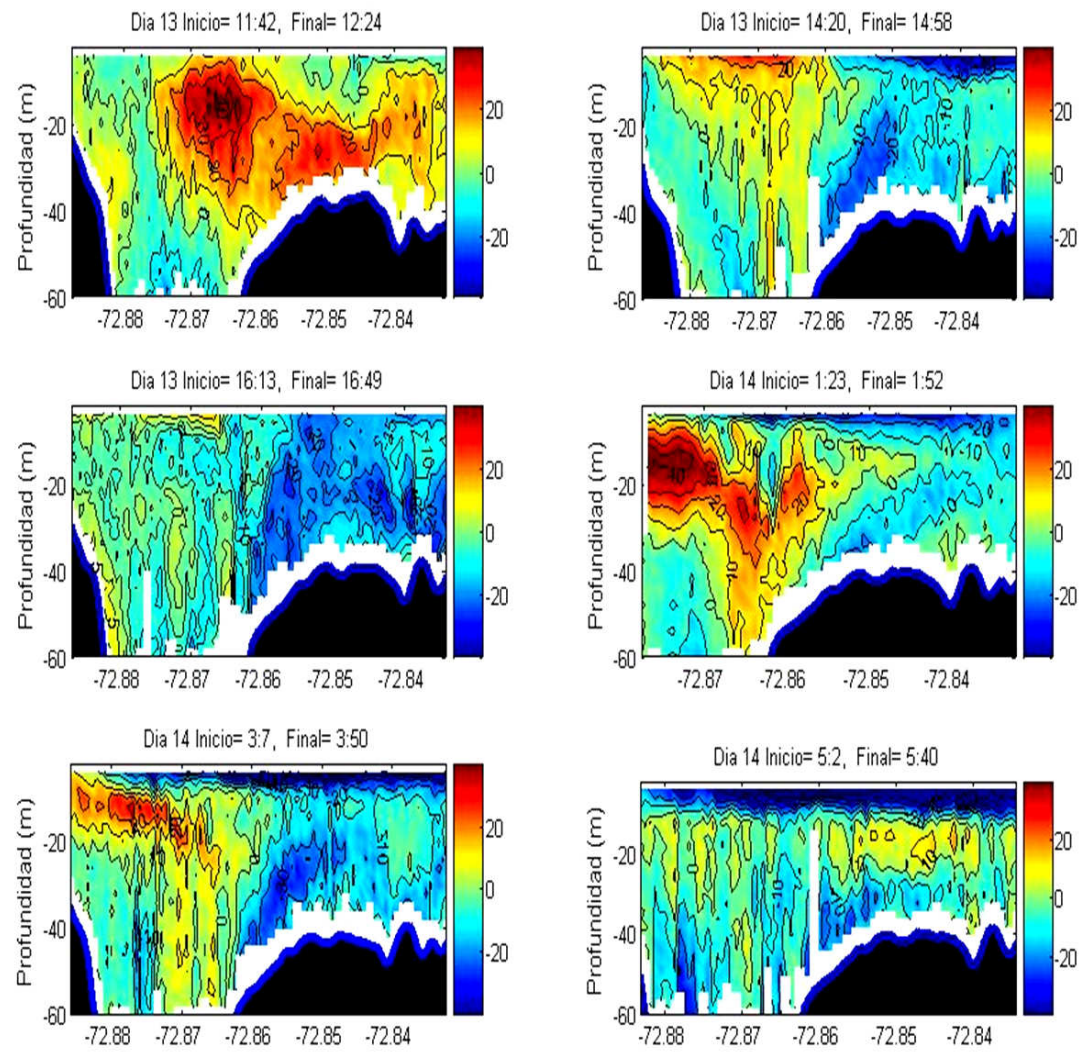

FIGURA 7. Distribución vertical de flujo en la dirección norte-sur medido por el ADCP de los 6 recorrido por el transecto 1. El fondo de color azul indica que la dirección de la corriente es hacia el sur y el fondo de color rojo va hacia el norte (los contornos sólidos son cada $10 \mathrm{~cm} / \mathrm{s}$ ).

FIGURE 7. Vertical distribution of north-southward flow recorded by the ADCP after 6 passing tracks at transect 1 . The color code is blue (southward), and red (northward), solid line is plotted each $10 \mathrm{~cm} / \mathrm{s}$.

Modelo Empírico: aplica la estimación del oleaje mediante metodología paramétricas de Massel (1996), las cuales se basan en modelos empíricos/teóricos desarrollados para los grandes lagos de Canadá y Estados Unidos. La aplicación de estos modelos debe considerar las características geográficas de este tipo de cuencas (longitudes máximas de 50 a $100 \mathrm{~km}$ ) y la forma de la topografía, con el fin de evitar una posible sobreestimación de los valores de altura de ola.

El seno de Reloncaví presenta una forma casi redonda, sin presencia de un eje preferencial de importancia. Aun, así se ha escogido los ejes de mayor longitud relativa para la estimación, correspondientes al eje Norte-Sur. Aún cuando no se posee información de viento de detalle para esta posición, se eligió esta dirección ya que es la que probablemente permiten una mayor transmisión de energía desde el viento (Fig. 8). Parte de la información utilizada en este estudio esta basada en información recolectada en los anuarios estadísticos de la Dirección Meteorológica de Chile (aeropuerto del Tepual y en el aeródromo de Chaitén).

Para el desarrollo de este estudio, se considera la metodología estándar conforme al análisis de oleaje propuesto por el Centro de Investigación de Ingeniera Costera de la Armada de los EEUU (CERC, 1984). Se estiman las alturas y periodos de olas operacionales y de diseño y finalmente se realiza una comparación con datos de olas medidos localmente en el área de estudio 


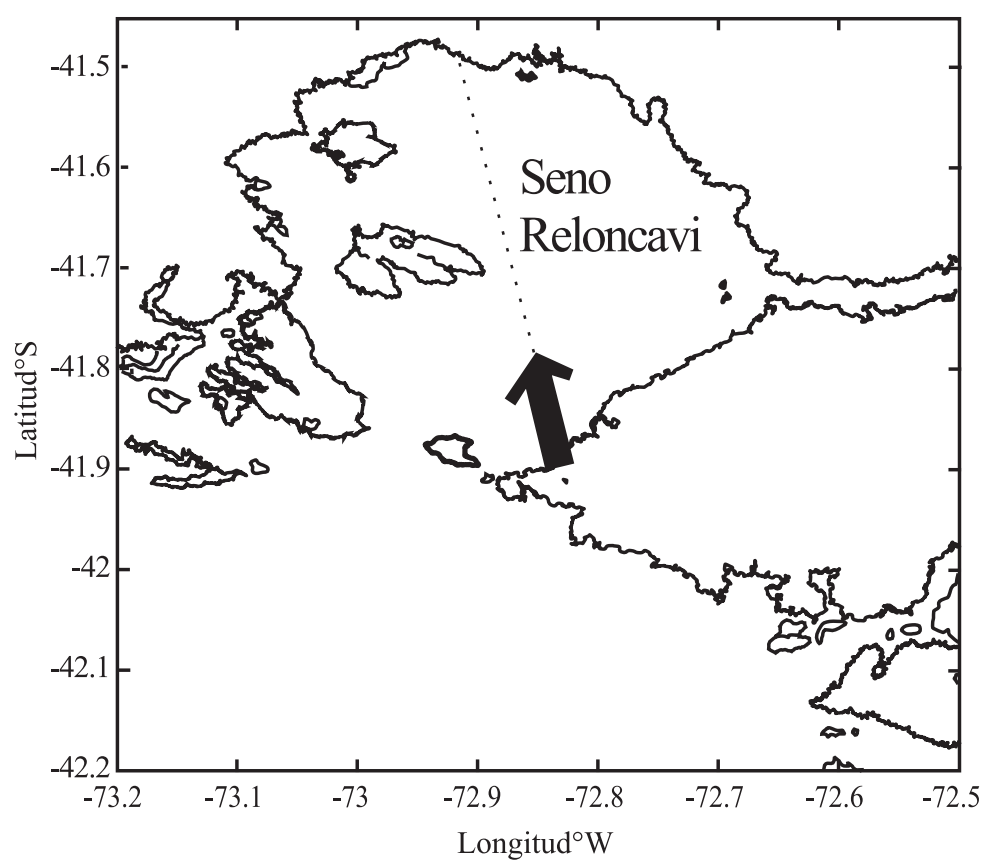

FIGURA 8.- Direcciones de viento predominante para el área de estudio.

FIGURE 8 . Wind predominant direction at the study area.

Se calcularon los Fetch disponibles para las direcciones de viento incidentes del SE y S que son los que afectarían en área de interés. Para el cálculo de la magnitud del Fetch se ha considerado las irregularidades del borde costero y la distintiva transferencia de energía del viento, en cada dirección radial. En efecto, el viento transmite energía no solo en la dirección que sopla, sino también lo hace radialmente en todas las direcciones de un arco de $\pm 15^{\circ}$. Para reunir información de oleaje en el área de interés, se efectúo una predicción de oleaje de generación local mediante retro-análisis basado en la información de viento disponible. Esta técnica consiste en realizar estimaciones de alturas y periodos de las olas para los escenarios meteorológicos extremos en función de los valores de vientos máximos registrados y las longitudes de Fetch efectivos. Esta metodología aparece descrita en CERC (1984) y Massel (1996). La figura 9 muestra los resultados, donde la ola alcanza la mayor altura, así, para un viento S-SE de $10.29 \mathrm{~m} / \mathrm{s}$ (20 nudos) y que sople 4,6 hrs el oleaje máximo es de $0,95 \mathrm{~m}$, mientras que en un caso extremo de viento de $30,87 \mathrm{~m} / \mathrm{s}$ (60 nudos) la ola alcanzaría 4,1 m después de 4,1 horas. Otros valores de oleaje para magnitudes típicamente observadas en la zona son de 0.4 y $0.67 \mathrm{~m}$.

Modelo Numérico: estimación del oleaje mediante modelo Mike-21 del Danish Hydraulic Institute, que resuelve la ecuación de Berkhoff en un ámbito espacial definido. Este simulador integra las ecuaciones dentro de un dominio rectangular que contiene la geometría tridimensional real del recinto de propagación. El modelo proporciona una solución estacionaria de la envolvente de alturas máximas de ola alcanzables en cada celda de la malla predefinida del recinto, para una determinada energía introducida en el sistema (estado de oleaje definido por los parámetros estadísticos representativos de altura de ola, periodo y dirección). Este modelo utiliza una batimetría de $2 \mathrm{Kms}$ de resolución y como forzantes usa datos de viento (componentes u y v) con resolución temporal de 3 horas y espacial de $50 \mathrm{Kms}$. Estas condiciones de viento son provenientes del modelo atmosférico global GFS (Global Forecast System) perteneciente a NCEP (National Center Environmental Prediction) de Estados Unidos. 


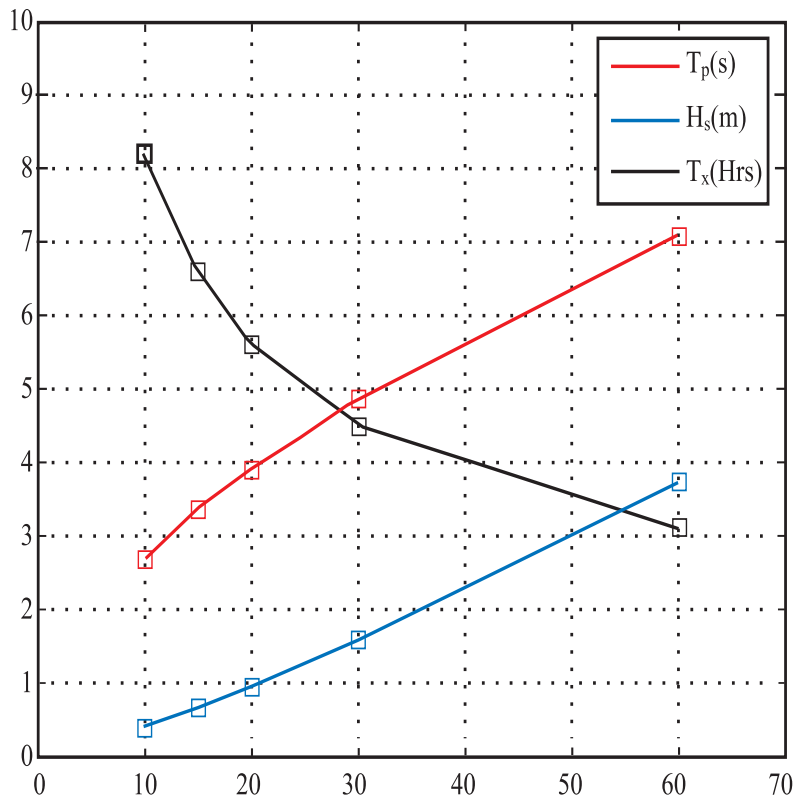

FIGURA 9. Valores de altura significativa $\left(\mathrm{H}_{\mathrm{sig}}\right)$, periodo medio $\left(\mathrm{T}_{\mathrm{p}}\right)$ y duración de viento $\left(\mathrm{t}_{\mathrm{x}}\right)$ para distintas velocidades de viento, Seno de Reloncaví.

FigURE 9. Significant height values (Hsig), average period (Tp), and wind duration (tx) for different wind velocities, Seno de Reloncaví.

Los siguientes esquemas de altura de olas fueron generados para dos casos típicos de condiciones atmosféricas y una condición extrema:

(1) Condición de baja presión (paso de un sistema frontal), vientos de dirección norte y noroeste y velocidades máximas que fluctúan entre los 50 y $60 \mathrm{~km} / \mathrm{h}$. Periodo de simulación de $60 \mathrm{hrs}$.

(2) Condición de alta presión, vientos de dirección sur y suroeste y velocidades máximas que fluctúan entre los 20 y $30 \mathrm{~km} / \mathrm{h}$. Periodo de simulación de 36 hrs.

(3) Condición extrema de alta presión (evento anómalo del 31 de diciembre de 2008), vientos de dirección sur y sureste y velocidades máximas que fluctúan entre los 40 y $50 \mathrm{~km} / \mathrm{h}$. Periodo de simulación de 96 horas.

La Tabla III muestra las 6 posiciones de las zonas donde se discutirá la altura de olas. Las estimaciones del modelo (Fig. 10) muestran que para una misma condición de viento (S) el desarrollo del oleaje es mayor en la boca del seno de Reloncaví alcanzando los $0,9 \mathrm{~m}$ al cabo de $25 \mathrm{hrs}$. Le siguen en importancia Quillaipe $(0,55 \mathrm{~m})$ y la boca del estuario Reloncaví $(0,55 \mathrm{~m})$. Estas zonas se caracterizan por la importancia de las corrientes que también fueron modeladas. Mientras que en las cercanías de Calbuco alcanza a $0,48 \mathrm{~m}$, en el paso de las islas Maillén y Guar solo llega a 0,43 m y Puerto Montt de 0,41 m. Estas últimas zonas están caracterizadas por la incidencia de corrientes de baja intensidad. El máximo secundario se observo en torno a las $17 \mathrm{hrs}$.

Dado el viento norte $(\mathrm{N})$, las estimaciones de las máximas alturas de oleaje (Fig 11), superaron a las generadas por el viento sur, aunque coinciden con que las máximas alturas que se apreciaron en la boca del seno $(1,69 \mathrm{~m})$. Pero, a diferencia de la anterior le siguen en importancia las cercanías de Calbuco (1,35 $\mathrm{m})$ y la boca del estero de Reloncaví Maillen y Guar $(0,79 \mathrm{~m})$ y Puerto Montt $(0,38 \mathrm{~m})$. Dos máximos secundarios de oleaje aparecieron en torno a las 13 hrs y 49 hrs. En ambos casos el desarrollo de las mayores alturas ocurre a las $04 \mathrm{hrs}$, aunque ocurre un máximo secundario anterior en torno a las $17 \mathrm{hrs}$ (viento sur) y $13 \mathrm{hrs}$ (viento norte). 
Gayana 73(1), 2009

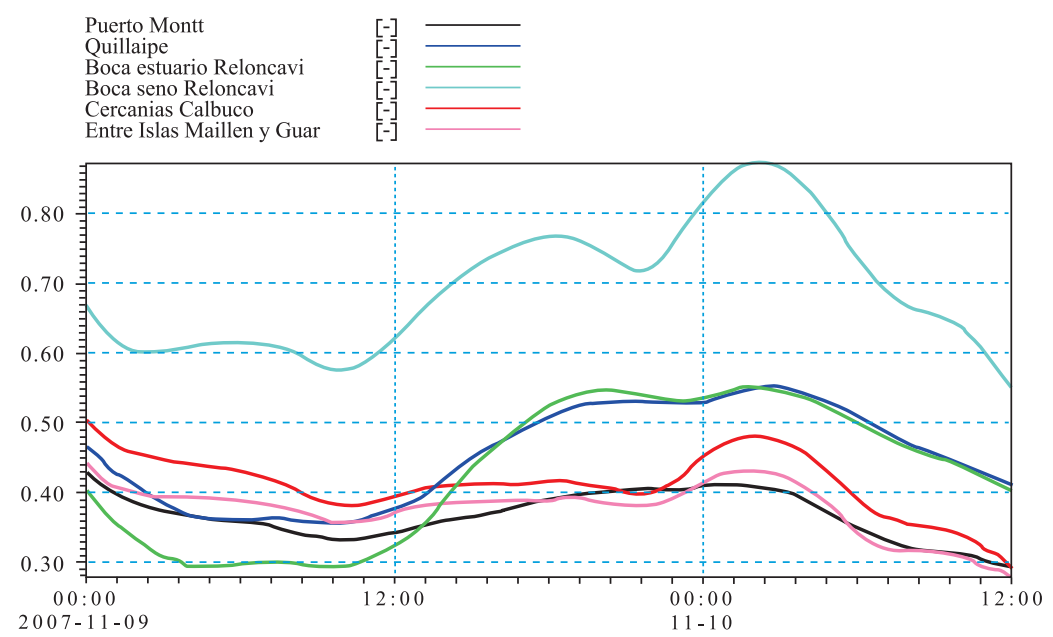

Figura 10. Ola significativa en el seno de Reloncaví con viento sur.

FIGURE 10. Significant wave in Seno de Reloncaví with southerly wind condition.

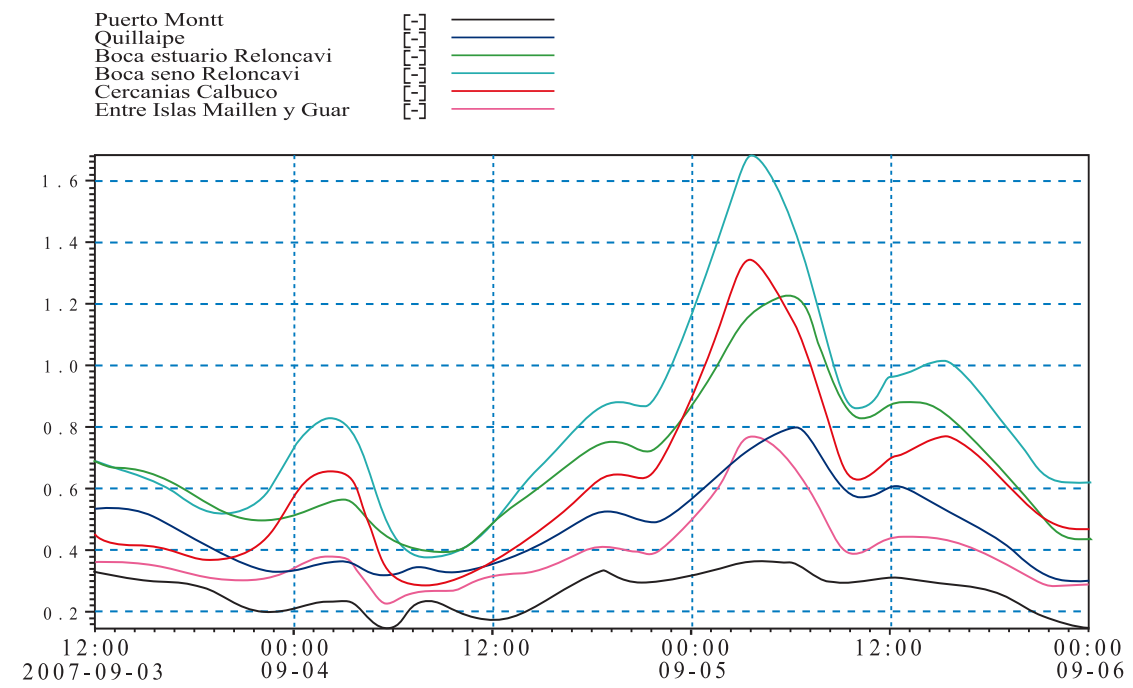

Figura 11. Ola significativa en el seno de Reloncaví con viento norte.

FIGURE 11. Significant wave in Seno de Reloncaví with northerly wind condition. 
La condición extrema (Fig. 12) de viento sur y sureste, al igual que en los casos anteriores, presenta la mayor altura de ola en la zona de la boca del seno Reloncaví (2.4 m), seguida por alturas muy similares en la zona de Puerto Montt y Quillaipe (2.2 m) finalmente las menores alturas de olas se registraron en los sectores de Maillen Guar y La boca del estuario con 1.6 y 1.75 m respectivamente. Cabe señalar que esta condición de viento provoca mayores alturas de olas que los anteriores esquemas. El desarrollo de las máximas alturas ocurre a las 24 horas.

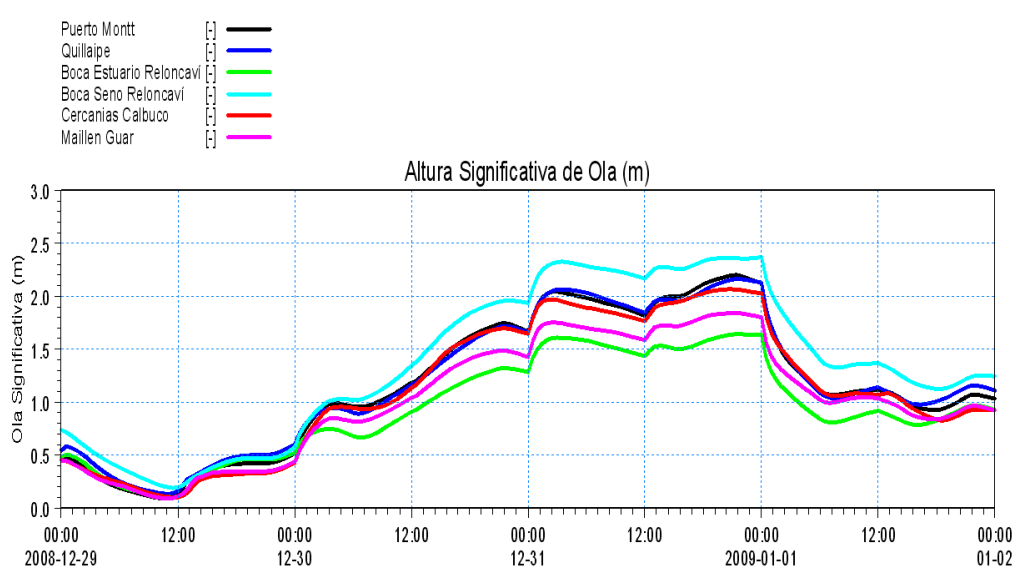

FIGURA 12. Ola significativa en el seno de Reloncaví con viento sur extremo.

FIGURE 12. Significant wave in Seno de Reloncavi with extreme southerly wind condition.

\section{DISCUSION Y CONCLUSION}

Este trabajo ha tenido como objetivo la recolección de datos disponibles de parámetros oceanográficos y atmosféricos, a partir de la literatura científica y de las empresas instaladas en la zona. La limitada información disponible (Tabla I) permite establecer que el seno Reloncaví es un sistema del tipo estuarino ya que esta principalmente formado por dos capas tal como se observa en las distribuciones verticales de temperatura, salinidad y oxigeno (Silva y Palma, 2006) influenciado por la pluma formada por la descarga del estero Reloncaví. La pluma somera ( 10 m) proveniente del Estuario Reloncaví juega un rol muy importante en la dinámica superficial y la cual es confirmada por la distribución vertical de corriente a través del Paso Nao y en los correntómetros de Chaicas y Quillaipe (5 $\mathrm{m}$ de profundidad).

La estación de Puerto Montt muestra a través de datos históricos que la máxima amplitud del nivel del mar es de $7.64 \mathrm{~m}$ con una amplitud promedio de $7.46 \mathrm{~m}$, superando la mayor parte de la costa de Chile evidenciando que la mayor parte de la variabilidad del nivel del mar esta asociada a la marea influenciando el sistema de corriente de la zona. En esta zona el esfuerzo del viento sería el fenómeno más importante para explicar la variabilidad de la circulación superficial y el oleaje. La topografía costera del seno Reloncaví afecta fuertemente la estructura de los vientos costeros y la circulación resultante del modelo. Para el caso de la estación meteorológica ubicada en Puerto Montt, la serie de dos años presentó una alta variabilidad, con fluctuaciones diarias, semanales y estacionales. La estacionalidad de la componente norte-sur se observa en el dominio del viento norte y noroeste entre abril y noviembre, y el dominio de la componente desde el sur entre noviembre y abril. 
TABLA III. Ubicación geográfica de las estaciones.

TABLE III. Geographical position of stations.

\begin{tabular}{lcc}
\hline Estación & Ubicación geográfica & Lugar de referencia \\
\hline 1 & $41^{\circ} 31^{\prime} \mathrm{S}, 72^{\circ} 54^{\prime} \mathrm{W}$ & Pto. Montt \\
2 & $41^{\circ} 37^{\prime} \mathrm{S}, 72^{\circ} 43^{\prime} \mathrm{W}$ & Ba. Lenca \\
3 & $41^{\circ} 43^{\prime} \mathrm{S}, 72^{\circ} 40^{\prime} \mathrm{W}$ & Boca Estero \\
4 & $41^{\circ} 50^{\prime} \mathrm{S}, 72^{\circ} 54^{\prime} \mathrm{W}$ & I. Queullín \\
5 & $41^{\circ} 44^{\prime} \mathrm{S}, 73^{\circ} 00^{\prime} \mathrm{W}$ & I. Guar \\
6 & $41^{\circ} 37^{\prime} \mathrm{S}, 73^{\circ} 00^{\prime} \mathrm{W}$ & I. Maillén \\
\hline
\end{tabular}

Las mediciones de corrientes obtenidas en las instalaciones de correntómetros de Chaicas y Quillaipe muestran para el periodo de otoño y primavera una alta variabilidad en magnitud y dirección. De igual forma, los resultados de corrientes obtenidos por el ADCP en el Paso Nao muestran con mayor resolución espacial la alta variabilidad del flujo en la vertical, y como en periodo de horas se observa un cambio radical en los patrones de corrientes. Aunque, persiste por el lado este del Paso Nao, una pluma superficial muy somera de de agua dulce pero de gran rapidez (superior a $20 \mathrm{~cm} / \mathrm{s}$ ) y corroboradas por las distribuciones verticales de temperatura y salinidad. Además, se observaron patrones comunes como la presencia de fluctuaciones diurnas y especialmente intensas las semidiurnas. Este parámetro dinámico (corriente directas) es uno de los resultados mas concluyentes que el seno Reloncaví es de una alta variabilidad y que los patrones de circulación cambian en periodos de horas.

La aplicación del método simple de parametrización de Massel entrega resultados de altura significativa de ola de $0,4 \mathrm{~m}$ para vientos intenso del sur de 10 nudos análogos a los valores que entrega el método numérico de Mike 21, pero con diferente tiempo de duración del viento. La altura de ola significativa en la zona protegida del mar interior de Chiloé estaría forzada principalmente por el viento local y en menor grado por el clima de ola oceánica y ola cercana (Caliskan \& Valle-Levinson, 2008). El clima de ola estaría siendo modificado por la gran variabilidad espacial y temporal de la magnitud y dirección de las corrientes de la onda de marea. Los resultados intermedios y no publicados también son consistentes para diferentes intensidades y constancia de los vientos. Esta coherencia en los resultados de estos cálculos independientes para la altura significativa de ola permite establecer que las aproximaciones generales son consistentes. Al respecto ambos tipos de estimación varían en el tiempo de desarrollo del oleaje, por lo que solamente mediciones en terreno pueden validar dichas estimaciones.

La limitada información disponible de la zona, solamente permite establecer aproximaciones a las características de circulación vertical y horizontal de la zona como también a la distribución espacial del campo de viento, las magnitudes del oleaje y los tiempos de retorno. Pero se estableció que la forma topográfica y batimetría de la zona, el intercambio por pasos estrechos con el Estero Reloncaví y al golfo de Ancud, la amplitud de la marea y de las corrientes de marea, como también la variabilidad del viento determinan el patrón de circulación y oleaje de la zona del seno de Reloncaví.

\section{AGRADECIMIENTOS}

Se agradece la colaboración de los señores Marcos Salamanca, Mario Cáceres y Aroldo Blaauboer que facilitaron diversos registros de mediciones. Este estudio se hizo con apoyo del proyecto CONICYTCORFO "Consorcio Tecnológico de Acuicultura en Zonas Expuestas en Chile"; Universidad de Concepción y Pontificia Universidad Católica de Valparaíso. 
Análisis oceanográficos y atmosféricos: Soto-MARDONES, L. ET AL.

\section{REFERENCIAS}

Borgel, R. 1970-1971. Geomorfología de las regiones australes de Chile Revista Geográfica de Chile Terra Australis. 21 (135-140).

Cáceres, M., A. Valle-levison, H. Sepúlveda \& K. HoLDERIED. 2002. Transverse variability of flow and density in a Chilean Fjord. Continental Shelf Research. 22: 1683-1698.

Cáceres, M., A. Valle-Levison \& L. Atkinson. 2003. Observations of cross-shannel structure of flow in an energetic tidal channel. Journal of Geophysical Research. 108 (C4): 11-1 - 11-9.

Cáceres M, A Valle-Levinson, J Fierro, M Bello \& M CAstiLlo. 2004. Variabilidad transversal del flujo submareal en fiordos del mar interior de Chile. En: CONA (ed). Crucero Cimar 9 Fiordos. Informes

Preliminares, PP. 25-35. Comité Oceanográfico Nacional, Valparaíso

Caliskan, H. \& A. valle-Levinson, 2008. Wind wave transformations in a elongated bay. Continental Shelf research 28: 1702-1710.

CERC, 1984. Shore Protection Manual. U.S. Army Coastal Engineering Research Center, Fort Belvoir,Vol. I y III. $2^{\circ}$ Edition.

Guzmán, D. y N. Silva. 2002. Caracterización física y química y masas de agua en los canales australes de Chile entre boca del Guafo y Golfo Elefantes (Crucero CIMAR 4 Fiordos). Cienc. Tecnol. Mar, 25(2): 45:76.

Massel, Stanislaw R. 1996. Ocean Surface Waves: Their Physics and Prediction.Advanced Series on Ocean Engineering. Vol 11.

PICKARD, G. 1971. Some physical oceanographic features of inlets of Chile. . Journal of Fisheries Researc Board of Canada 28. 8: 1077-1106.
RoDRIGo, C. 2006. Topografía submarina en canales de la Patagonia norte. En avances en el conocimiento oceanográficos de las aguas interiores chilenas, Puerto montt a cabo de Hornos N. Silva, \& S. Palma (Eds.). Comité Oceanográfico Nacional Pontificia Universidad Católica de Valparaíso, Valparaíso. $162 \mathrm{pp}$.

Silva, N., H. Sievers \& R. Prado. 1995. Descripción oceanográfica de los canales australes de Chile. Zona

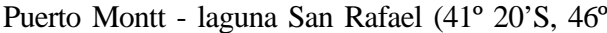
40’S) Revista de Biología Marína. 30 (2): 207-254.

Silva, N., C. Calvete y H. Sievers. 1998. Masas de agua y circulación general para algunos canales australes entre Puerto Montt y Laguna San Rafael, Chile (Crucero CIMAR 1 FIORDOS). Cienc. Tecnol. Mar, 21:17-48

Silva, N. \& S. Palma. 2006. El programa CIMAR en los canales y fiordos australes Avances en el conocimiento oceanográficos de las aguas interiores chilenas, Puerto Montt a cabo de Hornos N. Silva, \& S. Palma (Eds.). Comité Oceanográfico Nacional - Pontificia Universidad Católica de Valparaíso, Valparaíso, 162 pp.

Silva, N. \& S. Palma. 2006. Producción científica del Programa CIMAR en los canales y fiordos Australes. Cruceros CIMAR 1 a 4 Fiordos. pp. 145162. En: N. Silva \& S. Palma (eds.). Avances en el Conocimiento oceanográfico de las aguas interiores chilenas, Puerto Montt a cabo de Hornos. Comité Oceanográfico Nacional-Pontificia Universidad Católica de Valparaíso, 162 pp.

Valle-Levinson A., N. Sarkar, R. Sanay. D. Soto and J. LEÓN. Spatial structure of hidrography and flow in a Chilean fjord, Estuario Reloncavi. Estuaries and coasts, Vol. 30 (1): 113-126, 2007.

VERA, A. Efecto del viento sobre las variaciones del nivel del mar en el fiordo Aysén. Tesis Magister en oceanografía PUCV-UV. 57 pp. 2005.

Recibido: 04.02.09

Aceptado: 22.05.09 


\title{
ANALISIS DE PARAMETROS OCEANOGRAFICOS Y ATMOSFERICOS DEL SENO DE RELONCAVI
}

\section{ANALYSIS OF OCEANOGRAPHIC AND ATMOSPHERIC PARAMETERS OF SENO DE RELONCAVI}

\author{
Luis Soto-Mardones ${ }^{1}$, Jaime Letelier ${ }_{4}^{2,3}$ \\ Sergio Salinas ${ }^{2}$, E. Pinillas ${ }^{4}$ y Juan P. Belmar ${ }^{5}$ \\ 'Facultad de Ciencias, Departamento de Física, Universidad de Bío-Bío, \\ Av. Collao 1202, casilla 5-C, CP 4081112 Concepción, Chile. \\ ${ }^{2}$ Escuela de Ciencias del mar, Facultad de recursos naturales, \\ ${ }_{3}$ Pontificia Universidad de Católica de Valparaíso, Av. Altamirano 1480, Valparaíso, Chile. \\ ${ }^{3}$ Sección de Oceanografía Instituto de Fomento Pesquero, Av. Blanco 839, Valparaíso, Chile. \\ ${ }^{4}$ Sección de predicción del oleaje y la corrientes INTESAL. \\ ${ }^{5}$ Servicio Hidrográfico y Oceanográfico de la Armada, Errázuriz 254, Playa Ancha, Valparaíso.
}

\section{RESUMEN}

La recopilación de información histórica, tales como mediciones de corrientes, oleaje y viento no ha sido suficiente para describir la dinámica del seno Reloncaví. Sin embargo, a pesar de la información parcelada, las altas magnitudes de corrientes en las zonas de Chaica, Quillaipe y El Paso Nao nos permiten inducir que las corrientes baroclínicas modulan fuertemente la dinámica de la cuenca. Por otro lado, los vientos mostraron una alta variabilidad estacional y son modulados por la línea de costa, siendo estos en parte responsables de la circulación y el oleaje superficial. Además, la circulación superficial en los primeros $10 \mathrm{~m}$ esta fuertemente modulada por el ingreso de aguas dulce del estuario de Reloncaví. Además, la máxima amplitud del nivel del mar es de $7.64 \mathrm{~m}$, con una amplitud promedio de $7.46 \mathrm{~m}$, y donde la mayor parte de la variabilidad del nivel del mar esta asociada a la marea.

Finalmente, para describir el comportamiento del oleaje se comparan el método empírico de Massel con el modelo numérico del Mike21. Resultando que el clima de ola estaría siendo modificado por la gran variabilidad espacial y temporal de la magnitud y dirección de las corrientes de la onda de marea

Palabras claves: Baroclínicas, Olas, Pluma, Reloncaví, Viento

\section{ABSTRACT}

The historical information compilation of currents, waves, and wind measurements has not been enough to appropriately describe the dynamics of Seno de Reloncaví. However, in spite of the low diversity of studies, the high current magnitudes registered at Chaica, Quillaipe and Paso Nao allow us to infer baroclinic currents strongly modulate the basin dynamics. Furthermore, the wind showed a high seasonal variability, and it is modulated by the coastline, thus being somehow responsible of the surface circulation and waves. In addition, the surface circulation $(\sim 10 \mathrm{~m})$ is strongly modulated by freshwater input coming from Estuario de Reloncaví. Further, the maximum sea level amplitude was 7.64 m, with 7.46 $\mathrm{m}$ as an average, where the major sea level variability is linked to tide.

Finally, in order to describe the wave behavior, the results of the empiric method of Massel and the numerical model of Mike 21 are compared. As a result, the wave behavior would be modified by the high spatial and temporal variability of magnitude and direction of the tidal wave.

KEYwords: Baroclinic, Waves, Feather, Reloncavi, Wind 


\section{INTRODUCCION}

El seno de Reloncaví es un sistema semicerrado rodeado de una línea de costa casi circular que se conecta por el este con el estuario de Reloncaví y al sur con el Golfo de Ancud a través de tres pasos (Tautil, Queullin y Nao) que lo convierten en un laboratorio natural de mesoescala. El seno es el inicio de una de las mayores extensiones de canales, fiordos, senos y golfos interiores superando los $84000 \mathrm{~km}$ de costa en una extensión meridional de solo $1000 \mathrm{~km}$. Estas estructuras geográficas tienen su origen en la acción de la erosión glaciar y el hundimiento del valle central debido a la actividad tectónica de la zona (Borgel, 1970-1971). En su interior se desarrolla un sin número de sistemas estuarinos locales producto de la interacción de aguas salinas oceánicas y aguas dulces provenientes de aportes de lluvias, ríos, glaciares y ventisqueros (Silva et a., 1998; Guzmán y Silva, 2002; Cáceres et al., 2002; Cáceres et al., 2003; Cáceres, 2004;
Vera, 2005; Silva \& Palma, 2006; Valle-Levinson et al., 2007). Todos estos factores determinan que la dinámica de las corrientes de la zona de los canales este modulada por la influencia de la marea, los flujos salientes y entrantes desde los estuarios y fiordos (capa superior e inferior, respectivamente), como también desde los canales y pasos hacia el océano. Por otro lado, el oleaje en el mar interior estaría controlado por condiciones locales más que por perturbaciones oceánicas que pudieran entrar por los grandes canales que conectan con el océano como el canal Chacao y la boca del Guafo.

El presente trabajo consistió fundamentalmente en la recolección y análisis de datos disponibles de parámetros batimétricos, oceanográficos y atmosféricos, a partir de la literatura científica, instituciones y empresas que han realizado mediciones en la zona. A pesar de la escasa información disponible, es posible describir a grandes rasgos la dinámica que domina seno de Reloncaví.

TABLA I. Recolección de Parámetros Topográficos, Oceanográficos y Atmosféricos.

TABLE I. Topographic, oceanographic and atmospheric parameters compilation.

\begin{tabular}{|c|c|c|c|c|}
\hline \multirow[t]{2}{*}{ Parámetros } & \multicolumn{2}{|c|}{ Localización } & \multirow[t]{2}{*}{ Periodos } & \multirow[t]{2}{*}{ Fuentes } \\
\hline & Latitud (S) & Longitud W) & & \\
\hline Batimetría & $41^{\circ} 26^{\prime}-42^{\circ}$ & $73^{\circ} 45^{\prime}-72^{\circ} 30^{\prime}$ & & CIMAR \\
\hline Temperatura & \multirow{3}{*}{\multicolumn{2}{|c|}{$\begin{array}{l}\text { Estaciones } 1 \text { a } 4, \\
\text { dentro del seno } \\
\text { (Silva y Palma, 2006) }\end{array}$}} & $1 / 3 / 70-30 / 1 / 70$ & CIMAR \\
\hline Salinidad & & & $1 / 3 / 70-30 / 1 / 70$ & CIMAR \\
\hline Oxigeno & & & $1 / 3 / 70-30 / 1 / 70$ & CIMAR \\
\hline Nivel del Mar & $41^{\circ} 29^{\prime} 05^{\prime \prime}$ & $72^{\circ} 57^{\prime} 39^{\prime \prime}$ & 1990-2006 & SHOA \\
\hline Viento de Puerto Montt & $41^{\circ} 30^{\prime}$ & $72^{\circ} 54^{\prime}$ & $1 / 1 / 06-30 / 9 / 08$ & $\begin{array}{c}\text { Servicio Meteorológico de la } \\
\text { Armada }\end{array}$ \\
\hline Correntómetros Chaicas & $41^{\circ} 36^{\prime} 32^{\prime \prime}$ & $72^{\circ} 37^{\prime} 16^{\prime \prime}$ & $\begin{array}{l}20 / 3 / 06-5 / 5 / 06 \\
4 / 7 / 06-18 / 8 / 06\end{array}$ & Pesquera CAMANCHACA \\
\hline Correntómetros Quillaipe & $41^{\circ} 31^{\prime} 30^{\prime \prime}$ & $72^{\circ} 48^{\prime} 15^{\prime \prime}$ & $\begin{array}{l}\text { 20/3/06- } \\
5 / 5 / 06\end{array}$ & \\
\hline ADCP Paso Nao & $41^{\circ} 53,4^{\prime}$ & $72^{\circ} 49,2^{\prime}$ & $\begin{array}{l}11 / 10 / 04- \\
12 / 10 / 04\end{array}$ & CIMAR \\
\hline Modelo Empírico MASSEL & $41^{\circ} 26^{\prime}-42^{\circ}$ & $73^{\circ} 45^{\prime}-72^{\circ} 30^{\prime}$ & & \\
\hline Modelo Numérico MIKE21 & $41^{\circ} 26^{\prime}-42^{\circ}$ & $73^{\circ} 45^{\prime}-72^{\circ} 30^{\prime}$ & & INTESAL \\
\hline
\end{tabular}




\section{MATERIALES Y METODOS}

La información recopilada aparece detallada en el Tabla I, donde destaca la batimetría de alta resolución la cual fue compilada y grillada por Rodrigo (2006). Las distribuciones de parámetros oceanográficos de temperatura, salinidad y oxígeno de las zonas fueron reportadas por Pickard $(1971,1973)$ del crucero Hudson 1970. Además, se analizaron 17 años de datos diarios de nivel del mar de la estación mareográfica de Puerto Montt que fueron proporcionados por el Servicio Hidrográfico y Oceanográfico de la Armada (SHOA), mientras que el Servicio Meteorológico de la Armada proporcionó dos años de viento cada 3 horas de la estación Meteorológica de la misma ciudad. Por otro lado, la empresa Patagonia en Chaicas $\left(41^{\circ} 40^{\prime} \mathrm{S}-72^{\circ} 39^{\prime} \mathrm{W}\right)$ y Quillaipe $\left(41^{\circ} 34^{\prime} \mathrm{S}\right.$ - 72 $44^{\prime}$ W) instaló tres correntómetros en tres profundidades $(5,10$ y $20 \mathrm{~m})$ donde registró la magnitud y dirección de la corriente cada 5 minutos. La instalación de los correntómetros en Chaicas abarcó los periodos de otoño e invierno del 2006, mientras que las mediciones en Quillaipe abarcaron el periodo de otoño 2006. Además, se analizaron mediciones de corrientes realizadas con un equipo Acoustic Doppler Current Profiler (ADCP) en un circuito cerrado de 2 días de noviembre de 2004 en el Paso Nao, como parte del crucero Cimar Fiordo 10. Finalmente, se realizaron estimaciones de oleaje y corrientes mediante el modelo empírico de Massel y el modelo numérico Mike21 proporcionados por la empresa INTESAL.

\section{RESULTADOS}

Hidrografía: el transecto de temperatura, salinidad y oxígeno reportado por Silva et al., 1995 mediante un reanálisis de los trabajos de Pickard (1971, 1973), muestran claramente una pluma somera calida $\left(>13^{\circ} \mathrm{C}\right)$, de baja salinidad $(\sim 30 \%$ o) y de alta concentración de oxígeno (6 a $7.5 \mathrm{ml} / \mathrm{l})$. Los parámetros hidrográficos muestran una dinámica estuarina dominada por el aporte de agua dulce proveniente del estuario de Reloncaví, mientras que en profundidad es modulada por aguas de origen oceánica, es decir, la temperatura es menor a $11^{\circ} \mathrm{C}$, los índices de salinidad son superiores que en superficie (>32.5\%o) y el oxigeno es aproximadamente es menor $4 \mathrm{ml} / \mathrm{l}$.

Nivel del Mar: dentro del contexto de los forzantes de la circulación en la zona de los canales, la marea es uno de los factores más importantes en la zona. Los registros horarios y diarios del nivel del mar (NM) muestran una amplitud máxima de $7.64 \mathrm{~m}$ y una amplitud promedio de $7.46 \mathrm{~m}$ (Tabla II). Por otra parte, los resultados del NM diario (4.5 a 5.5 $\mathrm{m})$, con una amplitud máxima de $1 \mathrm{~m}$, permiten concluir que la mayor parte de la variabilidad en el registro de Puerto Montt está asociada principalmente a la marea. La máxima amplitud del NM aparece en dos fases anuales Marzo-Abril y Julio-Agosto. El promedio total de amplitud para un periodo de 15 año fue de $7.45 \mathrm{~m}$, mientras que la mínima amplitud $(7.26 \mathrm{~m})$ tiende a aparecer durante el mes de enero.

Tabla II. Estadística básica de valores de altura de nivel del mar y la amplitud en la estación costera de Puerto Montt (Fuente: Servicio Hidrográfico y Oceanográfico de la Armada de Chile).

Table II. Basic statistics of sea level, and amplitude at the coastal station of Puerto Montt (Source: Servicio Hidrográfico y Oceanográfico de la Armada de Chile).

\begin{tabular}{lccccccccccccc}
\hline $\mathbf{m})$ & Ene & Feb & Mar & Abr & May & Jun & Jul & Ago & Sep & Oct & Nov & Dic & Tot \\
\hline Promedio & 4.83 & 4.83 & 4.83 & 4.83 & 4.85 & 4.89 & 4.87 & 4.85 & 4.82 & 4.82 & 4.82 & 4.83 & 4.84 \\
Máximo & 8.64 & 8.61 & 8.59 & 8.80 & 8.58 & 8.51 & 8.51 & 8.63 & 8.48 & 8.64 & 8.53 & 8.53 & 8.80 \\
Mínimo & 1.38 & 1.15 & 1.07 & 1.18 & 1.33 & 1.12 & 0.94 & 1.01 & 1.00 & 1.15 & 1.20 & 1.20 & 0.94 \\
Amplitud & 7.26 & 7.46 & 7.52 & 7.62 & 7.25 & 7.39 & 7.57 & 7.62 & 7.48 & 7.49 & 7.33 & 7.33 & 7.45 \\
\hline
\end{tabular}


En términos relativos, el registro de nivel del mar muestra que las observaciones se concentraron $(>80 \%)$ entre $3.50 \mathrm{~m}$ y $6.50 \mathrm{~m}$ de altura. Por otro lado, menos de un $2.5 \%$ de las observaciones representan a observaciones del nivel del mar que sobrepasan los 8 $\mathrm{m}$, y estos corresponden a la combinación de las mareas más altas con la presencia de frentes de mal tiempo. En el dominio de las frecuencias de los datos horarios, la figura 1 muestra picos significativos asociados a las 24, 12, 6 y 4 hrs, resultando que la mayor parte de la variabilidad del NM está asociado a fluctuaciones de periodos menores a un día. Los períodos de 24 y $12 \mathrm{hrs}$ representan un régimen de marea semidiurno mixto, dos pleamares y dos bajamares diarias de significativa diferencia amplitud entre ellas. Los picos de 6 y 4 horas son componentes de agua somera de la marea que se potencian en aguas someras.

En el dominio de las frecuencias se determinó que la mayor parte de la variabilidad del NM está asociada a fluctuaciones de periodos menores a un día. Y la distribución general de valores extremos, indica que para un periodo de retorno de 10 años, la probabilidad es de un $10 \%$ y el NM es $\sim 8.62 \mathrm{~m}$; para un periodo de 20 años, la probabilidad de un 5 $\%$ es posible que ocurra un NM de $8.72 \mathrm{~m}$. Con una probabilidad de un $2 \%$ cada 50 años ocurre un NM de $8.77 \mathrm{~m}$ y con una probabilidad de $1 \%$ cada 100 años podría generarse un NM de $8.80 \mathrm{~m}$.

Viento: por las características topográficas del seno de Reloncaví la influencia de los vientos locales serían determinante en la estimación del oleaje y en la circulación superficial. En la estación meteorológica ubicada en Puerto Montt, el viento entre 2007 y 2008 presentó una alta variabilidad (Fig. 2), con fluctuaciones diarias, semanales y estacionales. La estacionalidad de la componente norte-sur (v) se observa claramente en el dominio del viento desde el norte, noroeste (abril y noviembre), y el dominio de la componente desde el sur (noviembre y abril). Además, en el dominio de las frecuencias de las direcciones del viento, se encontró que predominó en orden descendente: la combinación de viento sur $(i \%)$ y suroeste $(i \%)$, le sigue dirección norte $(i \%)$ y oeste $(i \%)$ entre un rango de $22 \%$ a $9 \%$ de los registros. Por otro lado, las magnitudes presentadas muestran que la mayor frecuencia de eventos de viento se concentró entre los rangos de 1 a $3 \mathrm{~m} / \mathrm{s}$ (40.9\%), de 3 a 6 m/s (34.6\%), mientras entre 6 a 9 $\mathrm{m} / \mathrm{s}$ se presentaron el $4 \%$ de los eventos y mas de 9 $\mathrm{m} / \mathrm{s}$ solamente el $0.16 \%$, mientras que calmas representan un $20.1 \%$ de los registros.

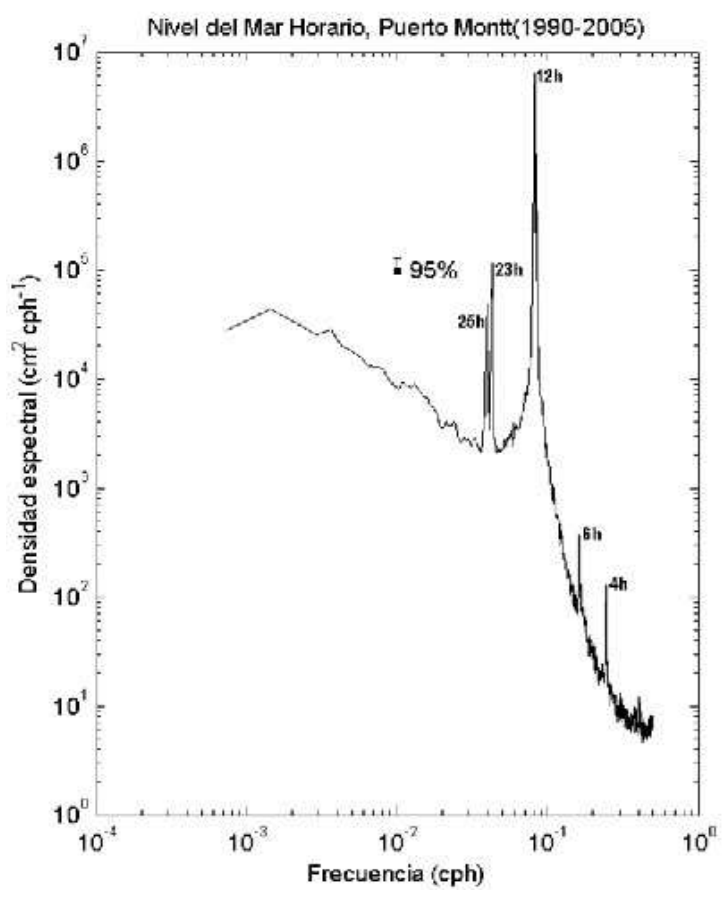

FIgURA 1. Espectro de frecuencia de datos de nivel del mar horarios de Puerto Montt que abarcan desde 1990 a 2005.

FIGURE 1. Frequency spectrum of hourly sea level data in Puerto Montt from 1990 up to 2005. 
Análisis oceanográficos y atmosféricos: Soto-MARDOnES, L. ET AL.
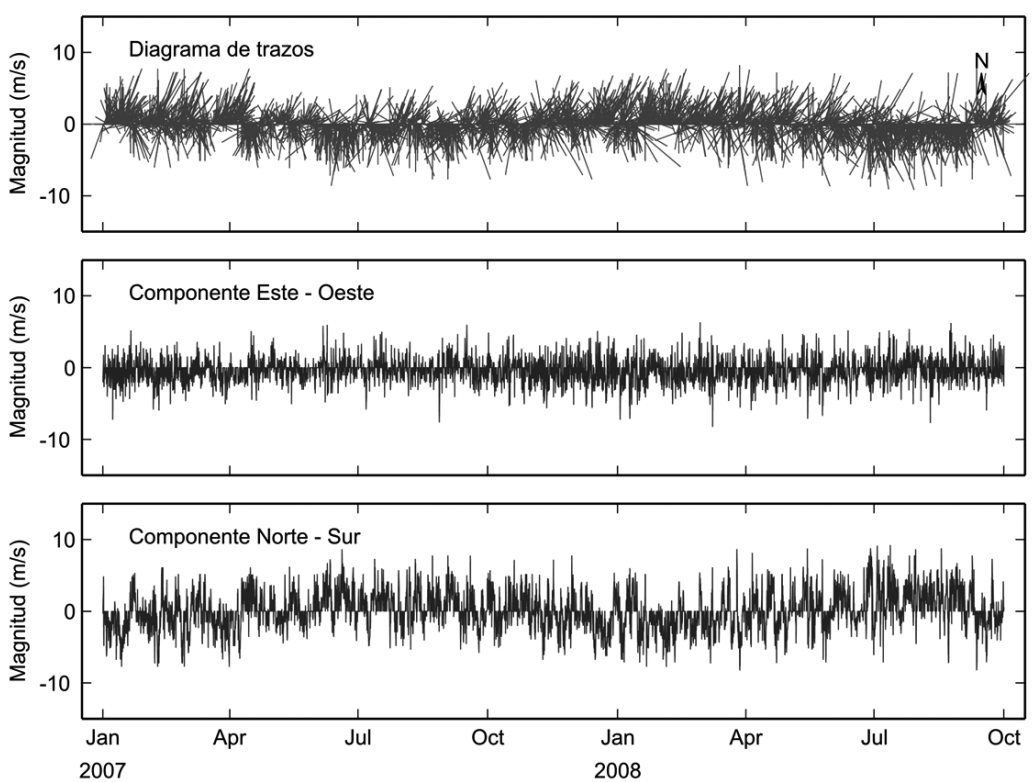

Figura 2. Diagrama de trazos y las componentes u (Este-Oeste) y v (Norte-Sur) del viento en Puerto Montt.

FIGURE 2. Vector plot and its wind components u (east-west) and v (north-south) in Puerto Montt.

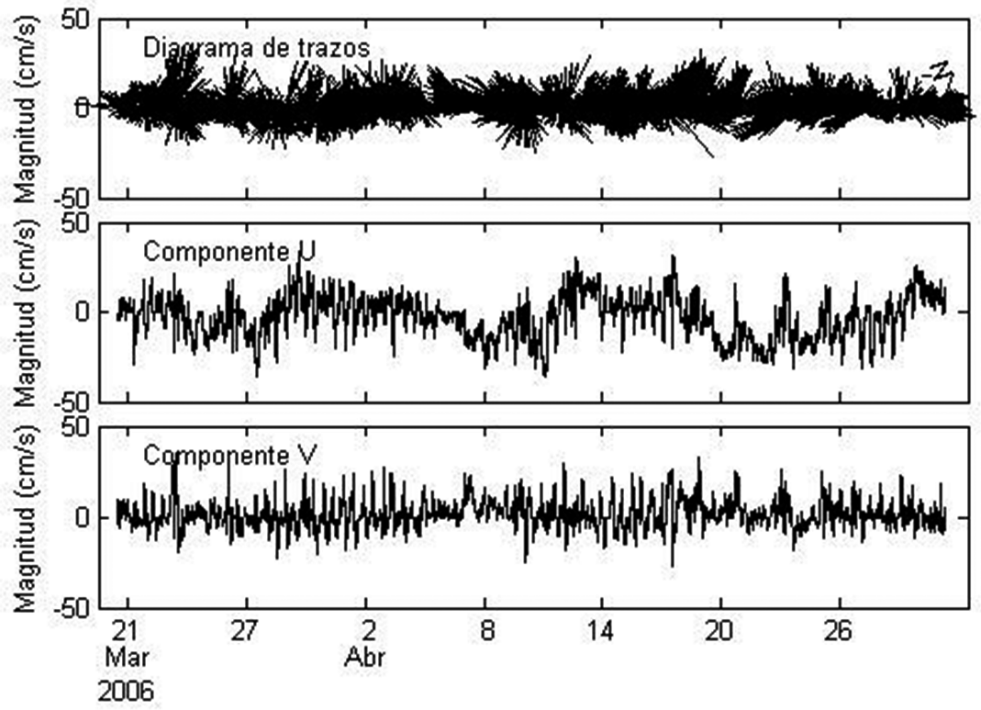

Figura 3. Diagrama de trazos y las componentes de velocidades U y V de instalación Chaicas (5m) para el periodo de otoño.

FIGURE 3. Vector plot and its velocity components $u$ and $v$ at Chaicas $(5 \mathrm{~m})$ for autumn season. 
Corriente: debido a las escasas mediciones de corrientes en el seno de Reloncaví, los anclajes de las estaciones de Chaicas y Quillaipe resultan una información valiosa. Las posiciones de los anclajes están indicadas en la Tabla I, cada instalación contiene tres correntómetros, ubicados en tres profundidades $(5,10$ y $20 \mathrm{~m})$. A $5 \mathrm{~m}$, se muestra una alta variabilidad mediante el diagrama de trazos y las componentes $\mathrm{U}$ y $\mathrm{V}$ fueron rotadas siguiendo el eje de máxima varianza $\left(\sim 70^{\circ}\right)$. Así, la máxima variabilidad es mostrada a lo largo (V) y perpendicular a la costa (U), donde las componentes presentan una señal diurna y semidiurna que varia en amplitud dependiendo del periodo del registro. La componente $\mathrm{U}$ presenta periodos de variación de entre 11 y 12 días. Además el máximo valor individual correspondió a $36,96 \mathrm{~cm} / \mathrm{s}$ en dirección NW.

A $10 \mathrm{~m}$ de profundidad, el instrumento presentó anomalías que no permitieron determinar la intensidad y dirección de las corrientes. Por otro lado, a $20 \mathrm{~m}$ de profundidad (Fig. 4), la intensidad de las corrientes es relativamente menor que en la capa superficial, además, las perturbaciones semidiurnas son mucho menores que las observadas en superficie. A pesar de lo anterior, aparece un evento importante que perturba la serie (entre el 17 y 22 de abril) con corrientes mayores a $10 \mathrm{~cm} / \mathrm{s}$ aparecen a lo largo y fuera de la costa en dirección noroeste (NW).

Las frecuencias en las direcciones de corriente mostró un flujo relativamente variable donde la dirección predominante fue la combinación $\mathrm{NW}(21,54 \%) \mathrm{y}$ $\mathrm{N}(14,91 \%)$ sobre las componentes $\mathrm{S}(13,05 \%)$ y $\mathrm{SE}$ $(11,71 \%)$ que la siguen en importancia.

El segundo conjunto de mediciones de la estación de Chaicas se realizó durante el período de invierno, cuyos resultados (Fig. 5) mostraron mayor intensidad y variabilidad que las corrientes observadas durante la época de otoño. De hecho, entre el 5 y 26 de julio, las intensidades sobrepasan los $20 \mathrm{~cm} / \mathrm{s}$ con pulsos cortos combinados de más de $40 \mathrm{~cm} / \mathrm{s}$ con dirección $\mathrm{S}$ y NW. Esta variabilidad se repite a partir de 6 de agosto donde predominaron flujos hacia fuera de la costa.

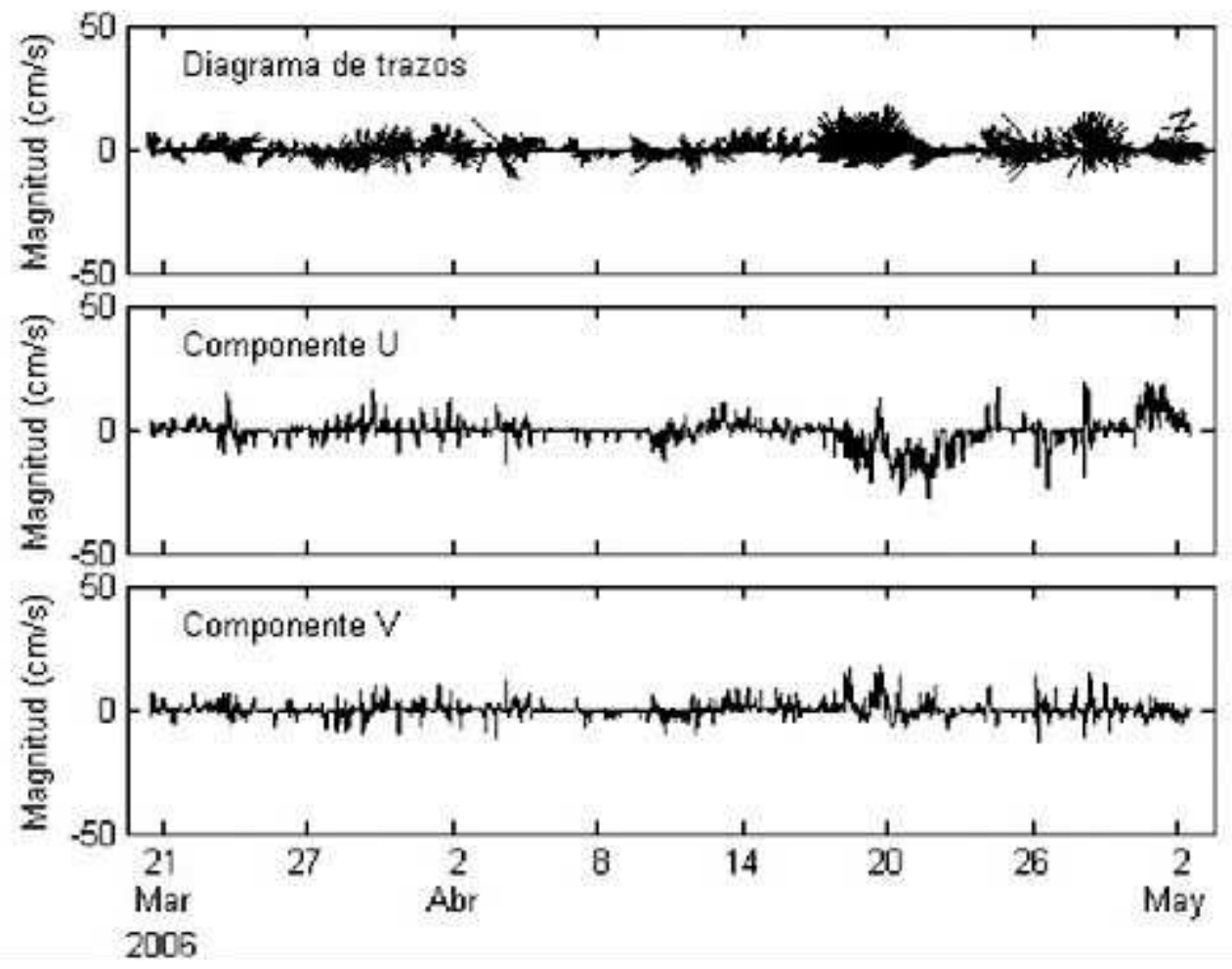

FIGURA 4. Diagrama de trazos y las componentes de velocidades U y V de instalación Chaicas (20m) para el periodo de otoño.

FIGURE 4 . Vector plot and its velocity components $u$ and $v$ at Chaicas $(20 \mathrm{~m})$ for autumn season. 


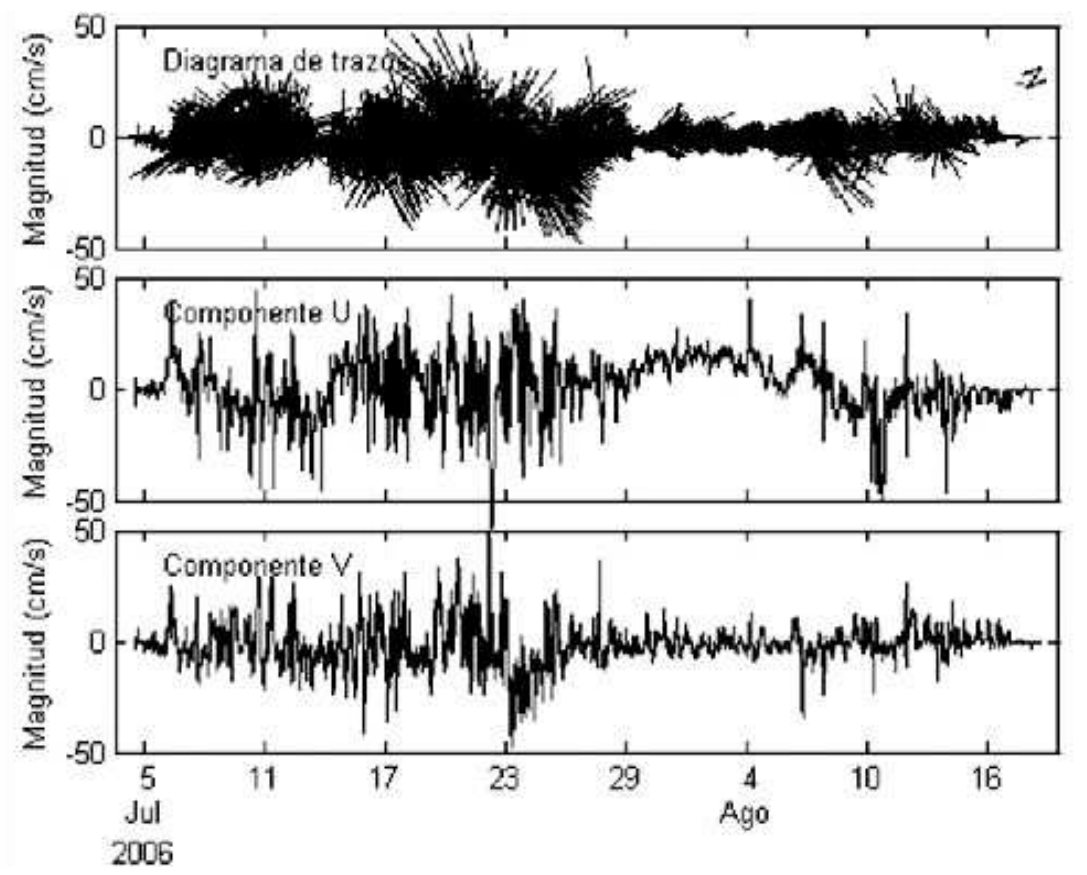

FIGURA 5. Diagrama de trazos y las componentes de velocidades U y V de instalación Chaicas (5m) para el periodo de invierno.

FIGURE 5. Vector plot, and its velocity components $\mathrm{u}$ and $\mathrm{v}(5 \mathrm{~m})$ for winter season.

Las frecuencias en las direcciones de las corrientes variaron desde S $(24,7 \%)$, SE $(163,15 \%)$ y NW (19.81\%) con el resto de las direcciones con porcentajes menores a $11 \%$. Los máximos individuales observados a esta profundidad superan los $63 \mathrm{~cm} / \mathrm{s}$ en dirección N y NW.

A $10 \mathrm{~m}$ de profundidad, los registros se observaron intensas corrientes $(>20 \mathrm{~cm} / \mathrm{s})$ con una fuerte componente perpendicular a la costa y una variabilidad en ambas componentes asociadas al ciclos diurnos y semidiurnos. Por otro lado, resaltan las corrientes a $20 \mathrm{~m}$ de profundidad, donde las magnitudes son superiores a las reportadas en otoño, con flujos significativos $(>15 \mathrm{~cm} / \mathrm{s})$ y pulsos de corrientes intensas $(>30 \mathrm{~cm} / \mathrm{s})$ a lo largo y perpendiculares a la costa.

Las mediciones realizadas en Quillaipe durante otoño muestran una gran variabilidad en dirección y magnitud. A la profundidad de $5 \mathrm{~m}$, las corrientes alcanzan magnitudes del orden de $10 \mathrm{c} 40 \mathrm{~cm} / \mathrm{s}$, con un promedio aproximado de $10 \mathrm{~cm} / \mathrm{s}$. A la profundidad de $10 \mathrm{~m}$ las corrientes son más débiles que en superficie y las máximas alcanzan magnitudes del orden de $\operatorname{los} 30 \mathrm{~cm} / \mathrm{s}$, con un promedio aproximado de $8 \mathrm{~cm} / \mathrm{s}$. Y finalmente, a la profundidad de $20 \mathrm{~m}$, las corrientes son relativamente más débiles que a $10 \mathrm{~m}$ de profundidad y los máximos registros alcanzan magnitudes del orden de los $25 \mathrm{~cm} / \mathrm{s}$, con un promedio aproximado de $8 \mathrm{~cm} / \mathrm{s}$. Las observaciones muestran que las corrientes en todas las profundidades en este sector son fuertemente afectadas por la onda de marea diurna y semidiurna. Resalta que las direcciones dominantes en las tres profundidades son hacia el NW $\left(320^{\circ}\right)$ y SE $\left(140^{\circ}\right)$. Otra información valiosa que se logró rescatar, son las mediciones realizadas con ADCP en el paso Nao, las que a pesar del corto periodo de muestreo (2 días), nos permite dar una visión global de la dinámica de la corrientes que atraviesan hacia el interior y exterior del seno, y permiten justificar la alta variabilidad que se observa en el campo de vientos, altura nivel del mar y los anclajes de Chaicas y Quillaipe. La figura 6 se muestra un circuito realizado cada tres horas por las pasadas por el transecto 


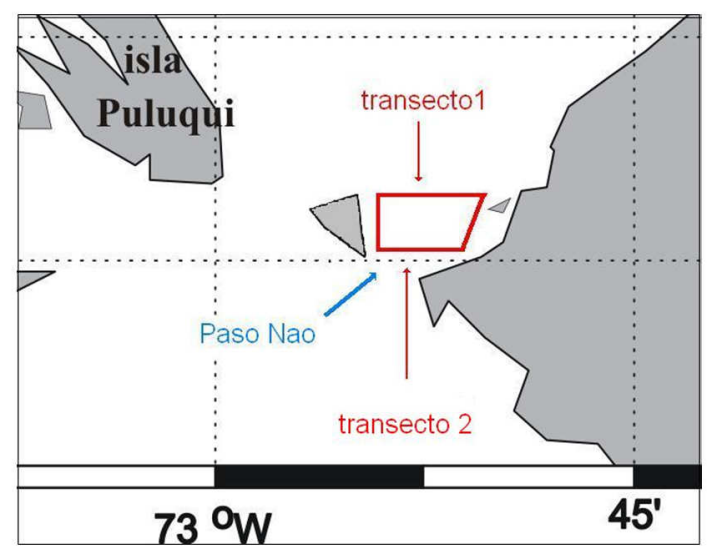

FIGURA 6. Muestreo con ADCP en el proyecto CIMAR 10 FIORDO, durante el periodo del 13 y 14 de noviembre del 2004.

FIGURE 6. ADCP measurements during CIMAR 10 FIORDO project, (November 13rd-14th, 2004).

Las figura 7 muestran las seis pasadas por el transecto 1 , donde se puede observar claramente en cada repetición del mismo recorrido ( $\sim 3 \mathrm{hrs})$ como el campo de distribución vertical de velocidad se va modificando. Por el borde oriental del Paso Nao, se observó una pluma somera (11:42 hrs) saliendo del seno de Reloncaví con velocidades de $10 \mathrm{~cm} / \mathrm{s}$ y un robusto núcleo se desplaza hacia el norte con velocidad sobre los $30 \mathrm{~cm} / \mathrm{s}$, posteriormente una columna hacia el sur desde la superficie hasta el fondo y una contracorriente pegada al borde occidental. $\mathrm{Al}$ cabo de tres horas (14:20 hrs) el esquema de circulación cambió radicalmente, donde el sector del borde occidental se desplazó hacia el norte y el borde oriental hacia el lado sur, además, se observó una intensificación de la pluma somera (> $30 \mathrm{~cm} / \mathrm{s})$. A las 16:13 hrs la circulación hacia el sur persistió, dominando casi toda el área del Paso Nao, posteriormente a las 01:23 del día 14 la circulación hacia el norte se fortaleció en el borde occidental alcanzando una velocidad en el centro de $40 \mathrm{~cm} / \mathrm{s}$, en cambio, en el borde oriental la circulación hacia el sur se presentó débil, aunque la pluma somera se expande hacia en oeste. A la 03:07 se observó una intensificación de la pluma somera alcanzando velocidades superiores a $30 \mathrm{~m} / \mathrm{s}$ en todo el ancho del paso Nao, mientras que la corriente hacia el norte - por el borde occidental- se debilitó ( $20 \mathrm{~cm} / \mathrm{s})$. A las 05:20 del segundo día, la circulación no mostró un patrón definido, aunque, persiste la intensificación de la pluma superficial (> $30 \mathrm{~cm} / \mathrm{s}$ )

Aunque no es posible comparar todos los parámetros (hidrografía, nivel del mar, viento, corrientes de anclajes) en forma temporal (Tabla I), queda en evidencia que estos confirman la alta variabilidad de la circulación superficial y en profundidad. Los datos de corrientes directas (Fig. 7) muestran en forma más convincente como las aguas del Seno de Reloncaví están fuertemente afectados por el forzante de la onda de marea y el desnivel de la superficie de agua causada por la acumulación de agua por efecto del viento que afecta a toda la columna de agua, por la circulación de tipo estuarino por el aporte de agua salobre proveniente del estuario de Reloncaví y por el efecto directo del viento sobre las capas superficiales de agua.

En este capitulo, de acuerdo a la recopilación de antecedentes, se analizan un modelo empírico y el otro numérico en ejecución en la zona de estudio. Para la aplicación de modelos numéricos en la cuenca semi-cerrada, somera y escasa influencia del océano abierto, las variables dinámicas que estarían modulando el oleaje serían: las corrientes marinas, marea, vientos y línea de costa. 

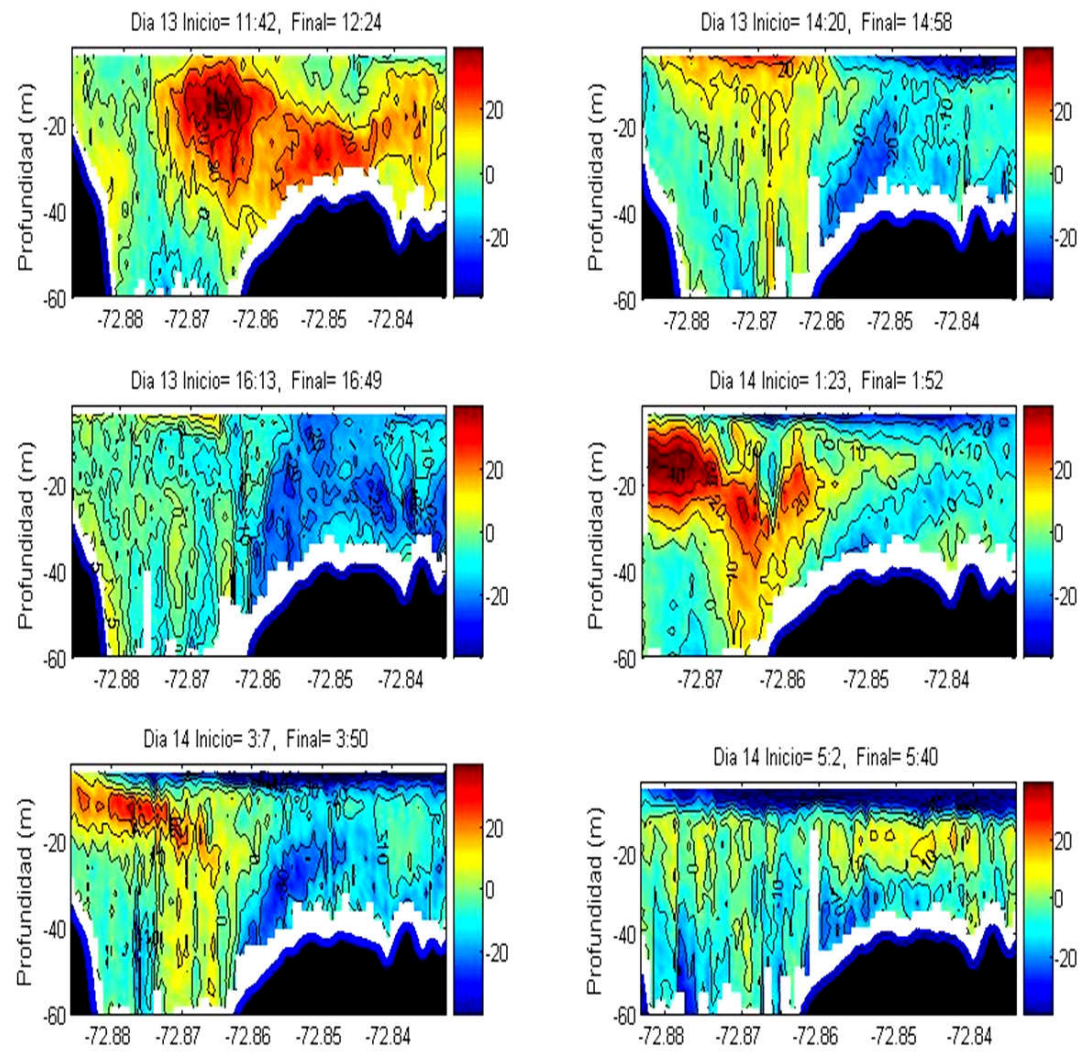

FIGURA 7. Distribución vertical de flujo en la dirección norte-sur medido por el ADCP de los 6 recorrido por el transecto 1. El fondo de color azul indica que la dirección de la corriente es hacia el sur y el fondo de color rojo va hacia el norte (los contornos sólidos son cada $10 \mathrm{~cm} / \mathrm{s}$ ).

FIGURE 7. Vertical distribution of north-southward flow recorded by the ADCP after 6 passing tracks at transect 1 . The color code is blue (southward), and red (northward), solid line is plotted each $10 \mathrm{~cm} / \mathrm{s}$.

Modelo Empírico: aplica la estimación del oleaje mediante metodología paramétricas de Massel (1996), las cuales se basan en modelos empíricos/teóricos desarrollados para los grandes lagos de Canadá y Estados Unidos. La aplicación de estos modelos debe considerar las características geográficas de este tipo de cuencas (longitudes máximas de 50 a $100 \mathrm{~km}$ ) y la forma de la topografía, con el fin de evitar una posible sobreestimación de los valores de altura de ola.

El seno de Reloncaví presenta una forma casi redonda, sin presencia de un eje preferencial de importancia. Aun, así se ha escogido los ejes de mayor longitud relativa para la estimación, correspondientes al eje Norte-Sur. Aún cuando no se posee información de viento de detalle para esta posición, se eligió esta dirección ya que es la que probablemente permiten una mayor transmisión de energía desde el viento (Fig. 8). Parte de la información utilizada en este estudio esta basada en información recolectada en los anuarios estadísticos de la Dirección Meteorológica de Chile (aeropuerto del Tepual y en el aeródromo de Chaitén).

Para el desarrollo de este estudio, se considera la metodología estándar conforme al análisis de oleaje propuesto por el Centro de Investigación de Ingeniera Costera de la Armada de los EEUU (CERC, 1984). Se estiman las alturas y periodos de olas operacionales y de diseño y finalmente se realiza una comparación con datos de olas medidos localmente en el área de estudio 


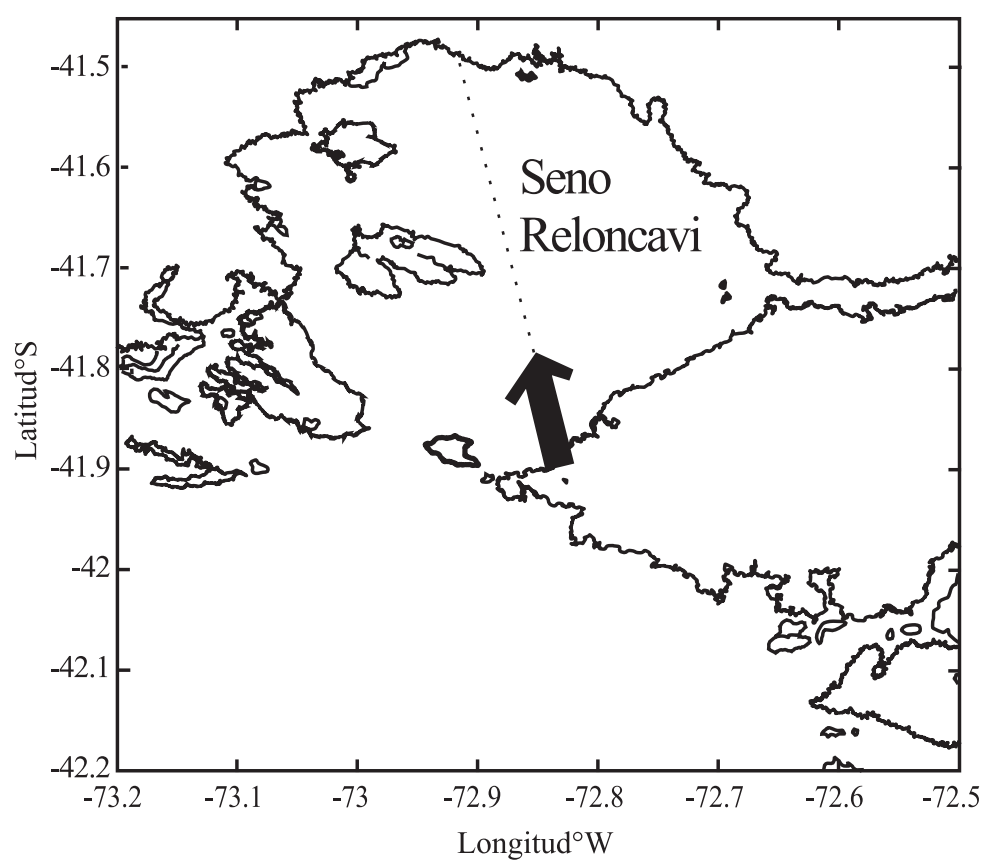

FIGURA 8.- Direcciones de viento predominante para el área de estudio.

FIGURE 8 . Wind predominant direction at the study area.

Se calcularon los Fetch disponibles para las direcciones de viento incidentes del SE y S que son los que afectarían en área de interés. Para el cálculo de la magnitud del Fetch se ha considerado las irregularidades del borde costero y la distintiva transferencia de energía del viento, en cada dirección radial. En efecto, el viento transmite energía no solo en la dirección que sopla, sino también lo hace radialmente en todas las direcciones de un arco de $\pm 15^{\circ}$. Para reunir información de oleaje en el área de interés, se efectúo una predicción de oleaje de generación local mediante retro-análisis basado en la información de viento disponible. Esta técnica consiste en realizar estimaciones de alturas y periodos de las olas para los escenarios meteorológicos extremos en función de los valores de vientos máximos registrados y las longitudes de Fetch efectivos. Esta metodología aparece descrita en CERC (1984) y Massel (1996). La figura 9 muestra los resultados, donde la ola alcanza la mayor altura, así, para un viento S-SE de $10.29 \mathrm{~m} / \mathrm{s}$ (20 nudos) y que sople 4,6 hrs el oleaje máximo es de $0,95 \mathrm{~m}$, mientras que en un caso extremo de viento de $30,87 \mathrm{~m} / \mathrm{s}$ (60 nudos) la ola alcanzaría 4,1 m después de 4,1 horas. Otros valores de oleaje para magnitudes típicamente observadas en la zona son de 0.4 y $0.67 \mathrm{~m}$.

Modelo Numérico: estimación del oleaje mediante modelo Mike-21 del Danish Hydraulic Institute, que resuelve la ecuación de Berkhoff en un ámbito espacial definido. Este simulador integra las ecuaciones dentro de un dominio rectangular que contiene la geometría tridimensional real del recinto de propagación. El modelo proporciona una solución estacionaria de la envolvente de alturas máximas de ola alcanzables en cada celda de la malla predefinida del recinto, para una determinada energía introducida en el sistema (estado de oleaje definido por los parámetros estadísticos representativos de altura de ola, periodo y dirección). Este modelo utiliza una batimetría de $2 \mathrm{Kms}$ de resolución y como forzantes usa datos de viento (componentes u y v) con resolución temporal de 3 horas y espacial de $50 \mathrm{Kms}$. Estas condiciones de viento son provenientes del modelo atmosférico global GFS (Global Forecast System) perteneciente a NCEP (National Center Environmental Prediction) de Estados Unidos. 


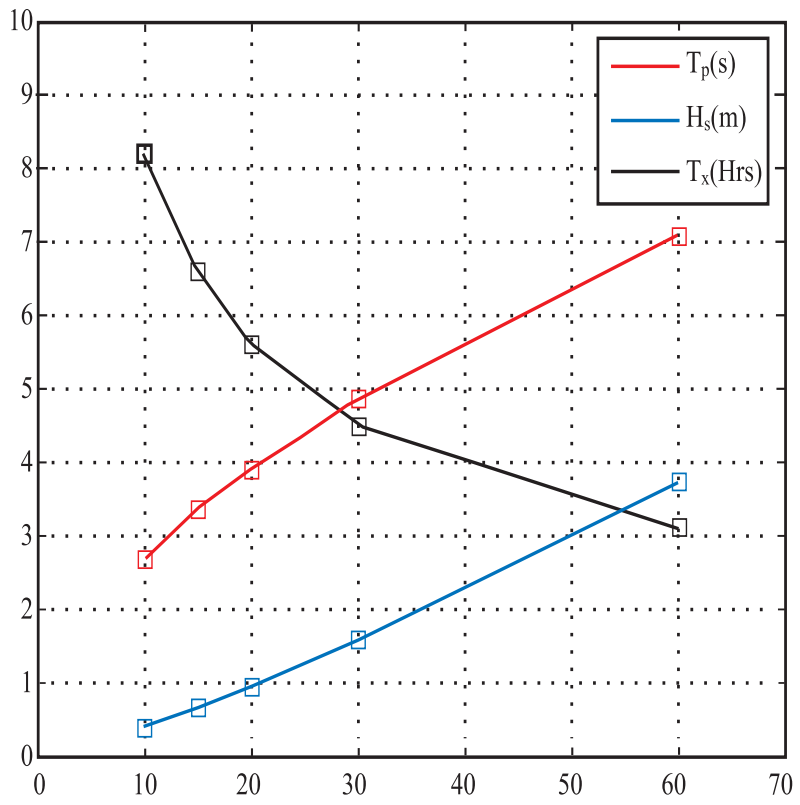

FIGURA 9. Valores de altura significativa $\left(\mathrm{H}_{\mathrm{sig}}\right)$, periodo medio $\left(\mathrm{T}_{\mathrm{p}}\right)$ y duración de viento $\left(\mathrm{t}_{\mathrm{x}}\right)$ para distintas velocidades de viento, Seno de Reloncaví.

FigURE 9. Significant height values (Hsig), average period (Tp), and wind duration (tx) for different wind velocities, Seno de Reloncaví.

Los siguientes esquemas de altura de olas fueron generados para dos casos típicos de condiciones atmosféricas y una condición extrema:

(1) Condición de baja presión (paso de un sistema frontal), vientos de dirección norte y noroeste y velocidades máximas que fluctúan entre los 50 y $60 \mathrm{~km} / \mathrm{h}$. Periodo de simulación de $60 \mathrm{hrs}$.

(2) Condición de alta presión, vientos de dirección sur y suroeste y velocidades máximas que fluctúan entre los 20 y $30 \mathrm{~km} / \mathrm{h}$. Periodo de simulación de 36 hrs.

(3) Condición extrema de alta presión (evento anómalo del 31 de diciembre de 2008), vientos de dirección sur y sureste y velocidades máximas que fluctúan entre los 40 y $50 \mathrm{~km} / \mathrm{h}$. Periodo de simulación de 96 horas.

La Tabla III muestra las 6 posiciones de las zonas donde se discutirá la altura de olas. Las estimaciones del modelo (Fig. 10) muestran que para una misma condición de viento (S) el desarrollo del oleaje es mayor en la boca del seno de Reloncaví alcanzando los $0,9 \mathrm{~m}$ al cabo de $25 \mathrm{hrs}$. Le siguen en importancia Quillaipe $(0,55 \mathrm{~m})$ y la boca del estuario Reloncaví $(0,55 \mathrm{~m})$. Estas zonas se caracterizan por la importancia de las corrientes que también fueron modeladas. Mientras que en las cercanías de Calbuco alcanza a $0,48 \mathrm{~m}$, en el paso de las islas Maillén y Guar solo llega a 0,43 m y Puerto Montt de 0,41 m. Estas últimas zonas están caracterizadas por la incidencia de corrientes de baja intensidad. El máximo secundario se observo en torno a las $17 \mathrm{hrs}$.

Dado el viento norte $(\mathrm{N})$, las estimaciones de las máximas alturas de oleaje (Fig 11), superaron a las generadas por el viento sur, aunque coinciden con que las máximas alturas que se apreciaron en la boca del seno $(1,69 \mathrm{~m})$. Pero, a diferencia de la anterior le siguen en importancia las cercanías de Calbuco (1,35 $\mathrm{m})$ y la boca del estero de Reloncaví Maillen y Guar $(0,79 \mathrm{~m})$ y Puerto Montt $(0,38 \mathrm{~m})$. Dos máximos secundarios de oleaje aparecieron en torno a las 13 hrs y 49 hrs. En ambos casos el desarrollo de las mayores alturas ocurre a las $04 \mathrm{hrs}$, aunque ocurre un máximo secundario anterior en torno a las $17 \mathrm{hrs}$ (viento sur) y $13 \mathrm{hrs}$ (viento norte). 
Gayana 73(1), 2009

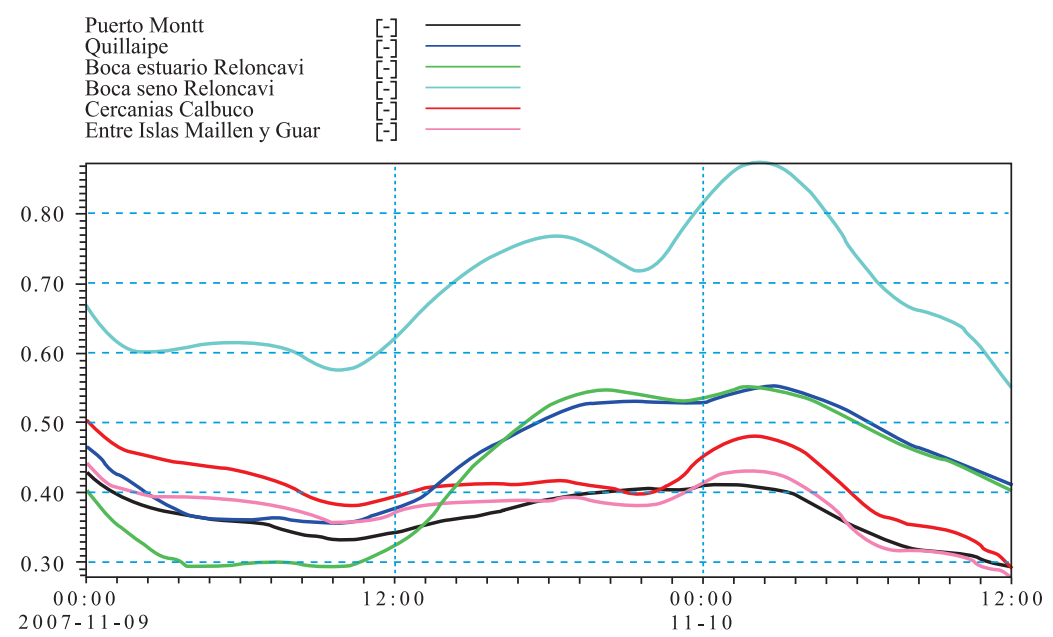

Figura 10. Ola significativa en el seno de Reloncaví con viento sur.

FIGURE 10. Significant wave in Seno de Reloncaví with southerly wind condition.

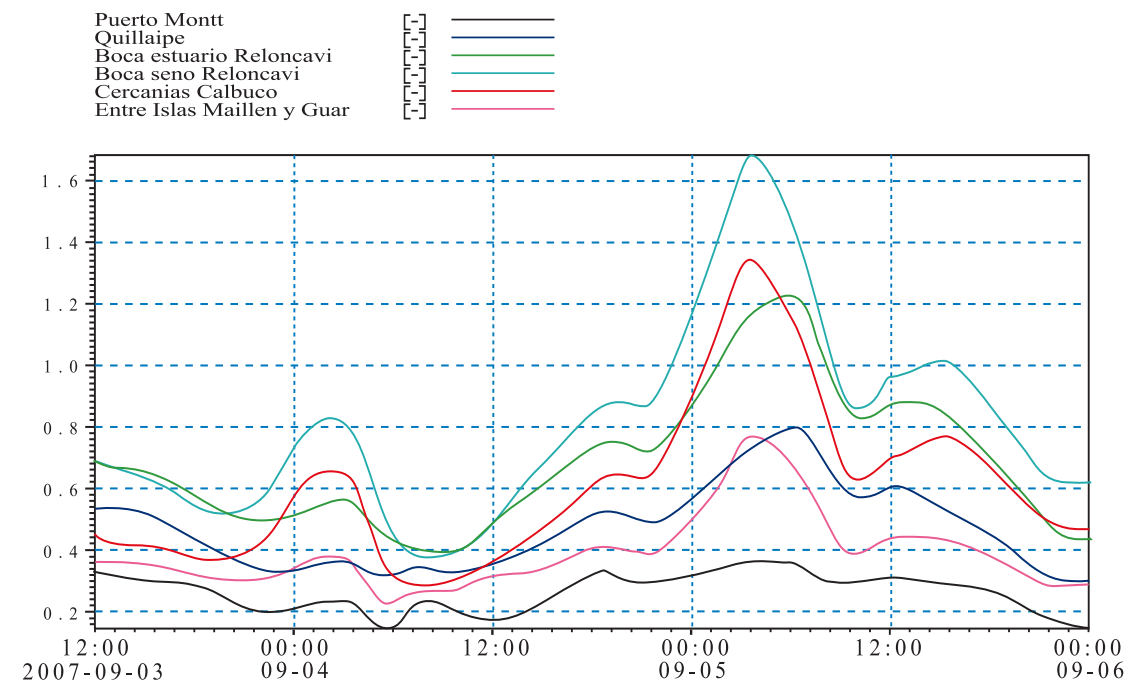

Figura 11. Ola significativa en el seno de Reloncaví con viento norte.

FIGURE 11. Significant wave in Seno de Reloncaví with northerly wind condition. 
La condición extrema (Fig. 12) de viento sur y sureste, al igual que en los casos anteriores, presenta la mayor altura de ola en la zona de la boca del seno Reloncaví (2.4 m), seguida por alturas muy similares en la zona de Puerto Montt y Quillaipe (2.2 m) finalmente las menores alturas de olas se registraron en los sectores de Maillen Guar y La boca del estuario con 1.6 y 1.75 m respectivamente. Cabe señalar que esta condición de viento provoca mayores alturas de olas que los anteriores esquemas. El desarrollo de las máximas alturas ocurre a las 24 horas.

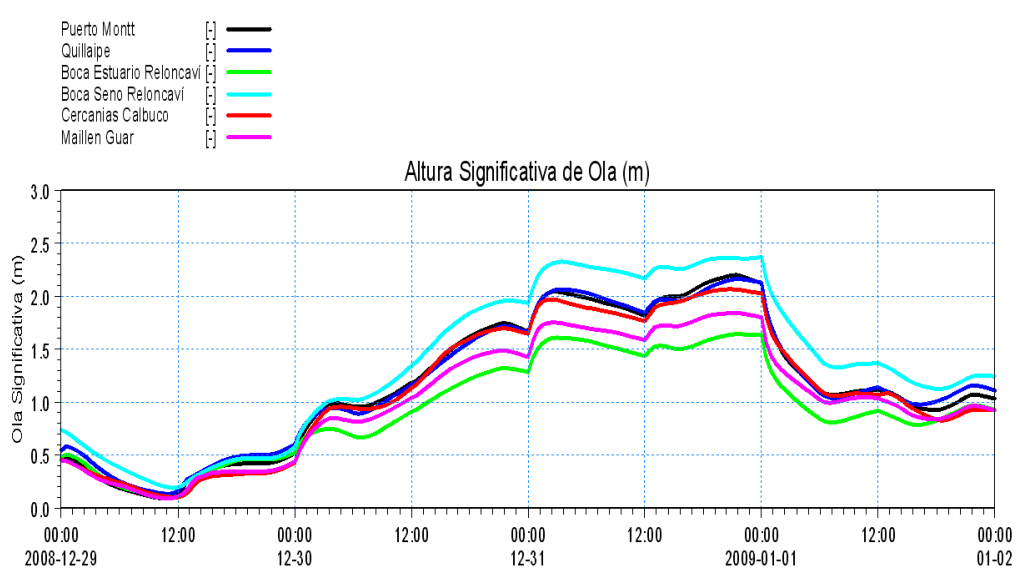

FIGURA 12. Ola significativa en el seno de Reloncaví con viento sur extremo.

FIGURE 12. Significant wave in Seno de Reloncavi with extreme southerly wind condition.

\section{DISCUSION Y CONCLUSION}

Este trabajo ha tenido como objetivo la recolección de datos disponibles de parámetros oceanográficos y atmosféricos, a partir de la literatura científica y de las empresas instaladas en la zona. La limitada información disponible (Tabla I) permite establecer que el seno Reloncaví es un sistema del tipo estuarino ya que esta principalmente formado por dos capas tal como se observa en las distribuciones verticales de temperatura, salinidad y oxigeno (Silva y Palma, 2006) influenciado por la pluma formada por la descarga del estero Reloncaví. La pluma somera ( 10 m) proveniente del Estuario Reloncaví juega un rol muy importante en la dinámica superficial y la cual es confirmada por la distribución vertical de corriente a través del Paso Nao y en los correntómetros de Chaicas y Quillaipe (5 $\mathrm{m}$ de profundidad).

La estación de Puerto Montt muestra a través de datos históricos que la máxima amplitud del nivel del mar es de $7.64 \mathrm{~m}$ con una amplitud promedio de $7.46 \mathrm{~m}$, superando la mayor parte de la costa de Chile evidenciando que la mayor parte de la variabilidad del nivel del mar esta asociada a la marea influenciando el sistema de corriente de la zona. En esta zona el esfuerzo del viento sería el fenómeno más importante para explicar la variabilidad de la circulación superficial y el oleaje. La topografía costera del seno Reloncaví afecta fuertemente la estructura de los vientos costeros y la circulación resultante del modelo. Para el caso de la estación meteorológica ubicada en Puerto Montt, la serie de dos años presentó una alta variabilidad, con fluctuaciones diarias, semanales y estacionales. La estacionalidad de la componente norte-sur se observa en el dominio del viento norte y noroeste entre abril y noviembre, y el dominio de la componente desde el sur entre noviembre y abril. 
TABLA III. Ubicación geográfica de las estaciones.

TABLE III. Geographical position of stations.

\begin{tabular}{lcc}
\hline Estación & Ubicación geográfica & Lugar de referencia \\
\hline 1 & $41^{\circ} 31^{\prime} \mathrm{S}, 72^{\circ} 54^{\prime} \mathrm{W}$ & Pto. Montt \\
2 & $41^{\circ} 37^{\prime} \mathrm{S}, 72^{\circ} 43^{\prime} \mathrm{W}$ & Ba. Lenca \\
3 & $41^{\circ} 43^{\prime} \mathrm{S}, 72^{\circ} 40^{\prime} \mathrm{W}$ & Boca Estero \\
4 & $41^{\circ} 50^{\prime} \mathrm{S}, 72^{\circ} 54^{\prime} \mathrm{W}$ & I. Queullín \\
5 & $41^{\circ} 44^{\prime} \mathrm{S}, 73^{\circ} 00^{\prime} \mathrm{W}$ & I. Guar \\
6 & $41^{\circ} 37^{\prime} \mathrm{S}, 73^{\circ} 00^{\prime} \mathrm{W}$ & I. Maillén \\
\hline
\end{tabular}

Las mediciones de corrientes obtenidas en las instalaciones de correntómetros de Chaicas y Quillaipe muestran para el periodo de otoño y primavera una alta variabilidad en magnitud y dirección. De igual forma, los resultados de corrientes obtenidos por el ADCP en el Paso Nao muestran con mayor resolución espacial la alta variabilidad del flujo en la vertical, y como en periodo de horas se observa un cambio radical en los patrones de corrientes. Aunque, persiste por el lado este del Paso Nao, una pluma superficial muy somera de de agua dulce pero de gran rapidez (superior a $20 \mathrm{~cm} / \mathrm{s}$ ) y corroboradas por las distribuciones verticales de temperatura y salinidad. Además, se observaron patrones comunes como la presencia de fluctuaciones diurnas y especialmente intensas las semidiurnas. Este parámetro dinámico (corriente directas) es uno de los resultados mas concluyentes que el seno Reloncaví es de una alta variabilidad y que los patrones de circulación cambian en periodos de horas.

La aplicación del método simple de parametrización de Massel entrega resultados de altura significativa de ola de $0,4 \mathrm{~m}$ para vientos intenso del sur de 10 nudos análogos a los valores que entrega el método numérico de Mike 21, pero con diferente tiempo de duración del viento. La altura de ola significativa en la zona protegida del mar interior de Chiloé estaría forzada principalmente por el viento local y en menor grado por el clima de ola oceánica y ola cercana (Caliskan \& Valle-Levinson, 2008). El clima de ola estaría siendo modificado por la gran variabilidad espacial y temporal de la magnitud y dirección de las corrientes de la onda de marea. Los resultados intermedios y no publicados también son consistentes para diferentes intensidades y constancia de los vientos. Esta coherencia en los resultados de estos cálculos independientes para la altura significativa de ola permite establecer que las aproximaciones generales son consistentes. Al respecto ambos tipos de estimación varían en el tiempo de desarrollo del oleaje, por lo que solamente mediciones en terreno pueden validar dichas estimaciones.

La limitada información disponible de la zona, solamente permite establecer aproximaciones a las características de circulación vertical y horizontal de la zona como también a la distribución espacial del campo de viento, las magnitudes del oleaje y los tiempos de retorno. Pero se estableció que la forma topográfica y batimetría de la zona, el intercambio por pasos estrechos con el Estero Reloncaví y al golfo de Ancud, la amplitud de la marea y de las corrientes de marea, como también la variabilidad del viento determinan el patrón de circulación y oleaje de la zona del seno de Reloncaví.

\section{AGRADECIMIENTOS}

Se agradece la colaboración de los señores Marcos Salamanca, Mario Cáceres y Aroldo Blaauboer que facilitaron diversos registros de mediciones. Este estudio se hizo con apoyo del proyecto CONICYTCORFO "Consorcio Tecnológico de Acuicultura en Zonas Expuestas en Chile"; Universidad de Concepción y Pontificia Universidad Católica de Valparaíso. 
Análisis oceanográficos y atmosféricos: Soto-MARDONES, L. ET AL.

\section{REFERENCIAS}

Borgel, R. 1970-1971. Geomorfología de las regiones australes de Chile Revista Geográfica de Chile Terra Australis. 21 (135-140).

Cáceres, M., A. Valle-levison, H. Sepúlveda \& K. HoLDERIED. 2002. Transverse variability of flow and density in a Chilean Fjord. Continental Shelf Research. 22: 1683-1698.

Cáceres, M., A. Valle-Levison \& L. Atkinson. 2003. Observations of cross-shannel structure of flow in an energetic tidal channel. Journal of Geophysical Research. 108 (C4): 11-1 - 11-9.

Cáceres M, A Valle-Levinson, J Fierro, M Bello \& M CAstiLlo. 2004. Variabilidad transversal del flujo submareal en fiordos del mar interior de Chile. En: CONA (ed). Crucero Cimar 9 Fiordos. Informes

Preliminares, PP. 25-35. Comité Oceanográfico Nacional, Valparaíso

Caliskan, H. \& A. valle-Levinson, 2008. Wind wave transformations in a elongated bay. Continental Shelf research 28: 1702-1710.

CERC, 1984. Shore Protection Manual. U.S. Army Coastal Engineering Research Center, Fort Belvoir,Vol. I y III. $2^{\circ}$ Edition.

Guzmán, D. y N. Silva. 2002. Caracterización física y química y masas de agua en los canales australes de Chile entre boca del Guafo y Golfo Elefantes (Crucero CIMAR 4 Fiordos). Cienc. Tecnol. Mar, 25(2): 45:76.

Massel, Stanislaw R. 1996. Ocean Surface Waves: Their Physics and Prediction.Advanced Series on Ocean Engineering. Vol 11.

PICKARD, G. 1971. Some physical oceanographic features of inlets of Chile. . Journal of Fisheries Researc Board of Canada 28. 8: 1077-1106.
RoDRIGo, C. 2006. Topografía submarina en canales de la Patagonia norte. En avances en el conocimiento oceanográficos de las aguas interiores chilenas, Puerto montt a cabo de Hornos N. Silva, \& S. Palma (Eds.). Comité Oceanográfico Nacional Pontificia Universidad Católica de Valparaíso, Valparaíso. $162 \mathrm{pp}$.

Silva, N., H. Sievers \& R. Prado. 1995. Descripción oceanográfica de los canales australes de Chile. Zona

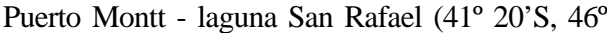
40’S) Revista de Biología Marína. 30 (2): 207-254.

Silva, N., C. Calvete y H. Sievers. 1998. Masas de agua y circulación general para algunos canales australes entre Puerto Montt y Laguna San Rafael, Chile (Crucero CIMAR 1 FIORDOS). Cienc. Tecnol. Mar, 21:17-48

Silva, N. \& S. Palma. 2006. El programa CIMAR en los canales y fiordos australes Avances en el conocimiento oceanográficos de las aguas interiores chilenas, Puerto Montt a cabo de Hornos N. Silva, \& S. Palma (Eds.). Comité Oceanográfico Nacional - Pontificia Universidad Católica de Valparaíso, Valparaíso, 162 pp.

Silva, N. \& S. Palma. 2006. Producción científica del Programa CIMAR en los canales y fiordos Australes. Cruceros CIMAR 1 a 4 Fiordos. pp. 145162. En: N. Silva \& S. Palma (eds.). Avances en el Conocimiento oceanográfico de las aguas interiores chilenas, Puerto Montt a cabo de Hornos. Comité Oceanográfico Nacional-Pontificia Universidad Católica de Valparaíso, 162 pp.

Valle-Levinson A., N. Sarkar, R. Sanay. D. Soto and J. LEÓN. Spatial structure of hidrography and flow in a Chilean fjord, Estuario Reloncavi. Estuaries and coasts, Vol. 30 (1): 113-126, 2007.

VERA, A. Efecto del viento sobre las variaciones del nivel del mar en el fiordo Aysén. Tesis Magister en oceanografía PUCV-UV. 57 pp. 2005.

Recibido: 04.02.09

Aceptado: 22.05.09 